\title{
Equilibrium securitization with diverse beliefs
}

\author{
ANDrew Ellis \\ Department of Economics, London School of Economics and Political Science \\ Michele Piccione \\ Department of Economics, London School of Economics and Political Science \\ SHENGXING ZHANG \\ Department of Economics, London School of Economics and Political Science
}

\begin{abstract}
We study the effects of diverse beliefs on equilibrium securitization under risk neutrality. We provide a simple characterization of the optimal securities. Pooling and tranching of assets emerges in equilibrium as a consequence of the traders' diverse beliefs about asset returns. The issuer of securities tranches the asset pool, and traders sort among the tranches according to their beliefs. We show how the traders' disagreement about the correlation of asset returns is a key factor in determining which assets are pooled.
\end{abstract}

KeYwords. Securitization, heterogeneous beliefs, collateral, tranching, pooling. JEL CLASSIFICATION. D53, G20.

\section{InTRODUCTION}

This paper investigates the implications of heterogeneous beliefs for the design of assetbacked securities and, in particular, the selection of the pool of assets backing them. We show that disagreement about the return of an asset provides an incentive to securitize that asset, and that disagreement about correlation between assets provides an incentive to pool them together. Disagreement about correlation has been pointed to by a number of authors, including Brunnermeier (2009), Coval et al. (2009), Hellwig (2009), Tett (2009), and Lewis (2010), as one of the main causes of the mispricing of securities in the run-up to the 2007-2008 financial crisis.

We begin by investigating security design in an economy that contains a safe asset and a single risky financial asset. The latter can be interpreted as an already formed pool of assets. A competitive issuer purchases units of the risky asset, and creates and sells asset-backed securities: promises to pay contingent on its return. The issuer can sell

Andrew Ellis: a.ellis@lse.ac.uk

Michele Piccione: m. piccione@lse.ac.uk

Shengxing Zhang: S. Zhang31@lse.ac.uk

We would like to thank Matt Darst, Darrell Duffie, Erik Eyster, Ana Fostel, Douglas Gale, John Geanakoplos, Filip Matejka, Balazs Szentes, Anton Tsoy, and conference/seminar participants at CERGE-EI, the Cowles Conference on General Equilibrium and its Applications, EUI, the EuroFIT workshop, the Hitotsubashi Summer Workshop, Manchester, Southampton, and Yale for helpful comments and discussion.

(C) 2022 The Authors. Licensed under the Creative Commons Attribution-NonCommercial License 4.0. Available at https://econtheory.org. https://doi.org/10.3982/TE4157 
any securities that are increasing in the risky asset's return, provided that its holdings are sufficient to cover all payments. Risk-neutral traders allocate their wealth between the safe asset, the risky asset, and securities; short-selling is prohibited.

We characterize a tight, central link between equilibrium securities and the traders' beliefs. The issuer tailors the securities to maximize disagreement about returns. Even if traders are not inherently optimistic or pessimistic about the risky asset's return, they may be so about particular securities. For instance, a low-variance trader is optimistic about debt but pessimistic about equity, and vice versa for a high-variance one. Because the issuer sells each security to the trader most optimistic about its return, creating disagreement increases revenue. We provide a straightforward yet powerful method to determine the optimal security design, and show how one can illustrate asset and security pricing, which securities are issued, and how the securities are allocated in a single, simple graph.

We embed our approach to security design into a general equilibrium model that permits the study of the interaction between the initial endowment distribution, the speculation that arises from heterogeneous beliefs, and asset prices. The equilibrium security design affects the trader's marginal utility of wealth and the value of her endowment, which in turn affects her willingness to pay for any given security. When the safe asset is scarce, these general equilibrium effects gain prominence, and the initial allocation of assets affects securitization and asset prices. We show that an equilibrium exists and that the consumption allocation is essentially unique. The issuer sells securities resembling tranches, each designed to appeal to a particular trader.

Next, we extend the model by endogenizing the issuer's asset pooling decision. There are two or more risky assets, and traders have diverse beliefs about the joint returns of these assets. The issuer purchases assets, allocates its purchases to pools, and then sells increasing securities, each based on the return of an individual pool. ${ }^{1}$

We define correlation agreement and disagreement in our model in terms of copulas. Traders agree about the correlation when they agree on the copula that maps the marginal distributions of assets into a joint distribution, and they disagree otherwise. This distinction is critical for the generation of asset pools in our model. The issuer benefits from pooling and tranching whenever traders disagree about the correlation, even if they agree on the individual return of every asset. In contrast, if traders disagree about the returns of individual assets but agree on their correlation, then pooling the assets is never profitable for the issuer. Intuitively, pooling assets increases disagreement when traders disagree about the correlation, but decreases it when they agree. As tranching exploits disagreement, pooling allows the issuer to profit from diverse beliefs about correlation.

These results emphasize correlation disagreement as a channel through which pooling can affect optimal security design. In contrast, most previous work focuses on pooling's ability to diversify away idiosyncratic risk and produce safe securities. This effect is absent in our model because of risk-neutrality. Turning off the diversification channel allows us to isolate the effect of correlation disagreement on securitization. In the

\footnotetext{
${ }^{1}$ Traders observe the content of the pools perfectly and know which pool backs each security.
} 
presence of other motives, incentives to exploit correlation disagreement by poolingand-tranching would still exist, though tempered by other considerations.

Belief diversity, and particularly correlation disagreement, is central to our analysis. As noted, correlation disagreement features prominently in the discussion of the role of securitization in the 2007-2008 financial crisis. Griffin et al. (2013, pp. 2272-2273) show that "there is considerable disagreement between Moody's and S\&P on the key assumptions-probabilities of default differ on average by $30 \%$ on the same [CDO] deal and default correlations [among assets in a given deal] differ by $57.2 \%$." More generally, the quality of statistical risk models used by financial intermediaries is varied and much lower than often assumed (Danielsson (2008)).

Evidence of correlation misperception, particularly neglect, has been found in several experimental studies, including Eyster and Weizsäcker (2010), Enke and Zimmermann (2017), and Rubinstein and Salant (2015). A recent literature studies the effects of correlation misperception, including DeMarzo et al. (2003), Ortoleva and Snowberg (2015), Levy and Razin (2015), and Ellis and Piccione (2017). Recent research has provided strong evidence for belief disagreement in financial markets more broadly. Greenwood and Shleifer (2014), Meeuwis et al. (2018), and Egan et al. (2020) all provide empirical evidence for belief heterogeneity across investors, and argue for its importance.

Our approach to equilibrium security design is more general than previous work. We do not restrict the number of securities or the types of beliefs (other than no atoms). Our characterization of the relationship between diverse beliefs and the endogenous emergence of tranches is related to the literature on collateralized lending with heterogeneous beliefs, which includes Geanakoplos (2001), Fostel and Geanakoplos $(2012,2015)$, Simsek (2013a), Geerolf (2015), and Gong and Phelan (2016) among others. While we put no restrictions on the number of monotone asset-backed securities and allow broad belief heterogeneity, the majority of these papers assume that securitization takes the form of collateralized debt (a senior tranche) and focus primarily on beliefs ranked by optimism and pessimism.

Our analysis of the incentives of issuers to select, prior to tranching, an asset pool when traders disagree about correlation is new. This topic has been overlooked in the recent literature, where most work addressing pooling, such as Broer (2018), compares equilibria when the pooling and tranching is taken as given. Notable exceptions include Bianchi and Jehiel (2018) and DeMarzo (2005). The former proposes a behavioral model where incorrect extrapolation from limited sampling gives an incentive to pool high and low quality assets together. The latter shows that selling debt, that is, a senior tranche, backed by a pool of independent assets can be the optimal security because it lessens adverse selection. In contrast, we show that tranching is the optimal securitization for inducing speculation on the correlation of the pool.

We contribute a simple yet general solution to the security design problem under diverse beliefs. Seminal work on optimal security design is due to Allen and Gale (1988), who do not focus on heterogeneous beliefs. However, diverse beliefs do feature in several recent papers on optimal security design. Garmaise (2001) studies optimal security design when securities are to be sold at auction, contrasting the equilibrium under "rational beliefs" with rational expectations. Simsek (2013b) considers the optimal creation 
of new assets for both speculation and risk-sharing purposes in a CARA-Normal general equilibrium model. Unlike our model, there are no collateral requirements for the designed securities, which must be linear combinations of random variables. Ortner and Schmalz (2016) study optimal security design by a strategic, optimistic issuer and a passive market with different beliefs about the return of a project. We restrict attention to increasing securities (unlike Allen and Gale (1988), Garmaise (2001), and Simsek (2013a)), and our analysis is general equilibrium (unlike Garmaise (2001) and Ortner and Schmalz (2016)).

A significant portion of the literature on securitization focuses on the role of asymmetric information. For instance, DeMarzo and Duffie (1999), DeMarzo (2005), Farhi and Tirole (2015) study optimal security design in an environment with asymmetric information about the asset quality and heterogeneity in traders' valuations. Under adverse selection, pooling and tranching produce liquidity that would be otherwise unavailable. In our model, traders have no private information. Pooling and tranching together create first moment disagreement even when none exists about individual assets in isolation.

The rest of the paper is organized as follows. Sections 2 and 3 present the benchmark model with a single risky asset and solve for the optimal security design. Section 4 explores the general equilibrium security design. Section 5 studies equilibrium with endogenous pooling of multiple risky assets.

\section{Model}

We consider an economy with one physical commodity, the consumption good, $N$ types of traders, labeled $i=1, \ldots, N$, and one representative firm, the issuer of asset-backed securities. There is a continuum of each type of trader having total measure one. ${ }^{2}$ The economy contains a risky asset, a unit of which delivers a random amount of the consumption good, and a safe asset, a unit of which delivers one unit of the consumption good. Trader $i$ is endowed with $e_{c}^{i}>0$ units of the safe asset, $e_{a}^{i} \geq 0$ units of the risky asset, and a share $\theta^{i} \geq 0$ of the firm. We assume $\sum_{i=1}^{N} e_{a}^{i}=e_{a}>0$, and that the shares in the firm sum to one, that is, $\sum_{i=1}^{N} \theta^{i}=1$. The risky asset delivers $s \in S \equiv[0, \bar{s}], \bar{s}>0$, units of the consumption good. ${ }^{3}$ Trader $i$ 's beliefs about $s$ are described by a nonatomic, cumulative distribution function $(\mathrm{CDF}), F^{i}(\cdot)$, or equivalently, the complementary CDF, $\widetilde{F}^{i}(\cdot) \equiv 1-F^{i}(\cdot)$, with support contained in $S$. For a real-valued measurable function $g: S \rightarrow \mathbb{R}$, let $\mathbb{E}^{i}[g]$ denote Trader $i$ 's expectation $\int g d F^{i}$. For notational simplicity, $\bar{s}$ is set to be the smallest state at which all complementary CDFs vanish. Each trader is risk neutral. ${ }^{4}$

The issuer can create financial contracts, or securities, backed by the asset. For standard reasons related to moral hazard (Innes 1990), we restrict attention to increasing

\footnotetext{
${ }^{2}$ The measure assigned to any type of trader is inessential.

${ }^{3}$ The set $S$ is endowed with the Borel $\sigma$-algebra.

${ }^{4}$ In an earlier version of this paper, Ellis et al. (2019), we allow for risk-aversion. We can alternatively interpret the CDF as the "risk-neutral CDF" that reflects both a trader's belief and exogenous state-dependent marginal utility.
} 
securities. Formally, the issuer can sell any security in the set

$$
\Sigma=\left\{\phi: S \rightarrow \mathbb{R}_{+} \mid \phi \text { is increasing }\right\}
$$

endowed with the supnorm. Denote the set of finite Borel measures on $\Sigma$ by $\mathcal{M}(\Sigma)$.

REMARK 1. State $s$ is the return of a risky asset that is potentially an already-formed asset pool. Any additional aggregate state is integrated out for simplicity, although the model easily extends to incorporate this additional uncertainty explicitly. While $s$ is potentially correlated with the aggregate state of the economy, it is still easily manipulable as long as the correlation is not perfect, at least within a small interval. By restricting securities to be increasing, any such manipulation is unprofitable; see Innes (1990), Nachman and Noe (1994), DeMarzo and Duffie (1999), and Biais and Mariotti (2005).

The issuer and all traders are price takers. Normalizing the price of the safe asset to unity, the risky asset sells at the price $p$ and each security $\phi \in \Sigma$ at the price $q(\phi)$. The security price function $q: \Sigma \rightarrow \mathbb{R}_{+}$is bounded and measurable with respect to the Borel $\sigma$-algebra on $\Sigma$. Short selling is ruled out.

The issuer maximizes its profit, buying the assets and selling securities. Since securities must be increasing, there is no incentive for the issuer to back securities with the safe asset in addition to the risky asset. Thus, the issuer purchases an amount $a^{0} \geq 0$ of the risky asset and supplies securities according to a measure $\mu^{0} \in \mathcal{M}(\Sigma)$. Its objective is to maximize profits

$$
\int_{\Sigma} q d \mu^{0}-p a^{0}
$$

subject to the securities being feasible:

$$
\int_{\Sigma} \phi(s) d \mu^{0} \leq s a^{0} \quad \text { for all } s \in S .
$$

Note that the securities produced must be fully backed by the asset. The issuer's profits are denoted by $\Pi$.

Remark 2. The assumption that the issuer attaches no value to any unsold portion of the assets, and thus has no belief of her own to value it, is not essential. If one allows for unsold portions of the assets and, for the same moral hazard reasons as for securities, imposes that the issuer's retained claims must be increasing, then the retained claims can be thought of as a security which should be traded on the market. That is, the trader who values the retained claims the most would want to purchase ownership of the firm to obtain them, which would fetch the same price as the corresponding security. Similarly, we could do away with the issuer entirely and allow traders to issue securities under these same restrictions without altering our analysis, though the issuer interpretation is more natural. 
Trader $i$ maximizes her expected utility by purchasing an amount $a^{i} \geq 0$ of the risky asset, holding an amount $c^{i} \geq 0$ of the safe asset, and purchasing securities. ${ }^{5}$ Her demand for securities is given by a measure $\mu^{i} \in \mathcal{M}(\Sigma)$. Thus, she maximizes

$$
\mathbb{E}^{i}\left[s a^{i}+\int_{\Sigma} \phi(s) d \mu^{i}+c^{i}\right]
$$

subject to the budget constraint

$$
p a^{i}+\int_{\Sigma} q d \mu^{i}+c^{i} \leq e_{c}^{i}+p e_{a}^{i}+\theta_{i} \Pi .
$$

An allocation $\left(a^{0}, \mu^{0} ;\left(a^{i}, c^{i}, \mu^{i}\right)_{i=1}^{N}\right)$ is attainable if $a^{0} \geq 0, a^{i}, c^{i} \geq 0, i=1, \ldots, N$, equation (2) holds, and

$$
\begin{aligned}
& \sum_{i=1}^{N} \mu^{i}=\mu^{0}, \\
& \sum_{i=0}^{N} a^{i}=\sum_{i=1}^{N} e_{a}^{i}, \\
& \sum_{i=1}^{N} c^{i}=\sum_{i=1}^{N} e_{c}^{i} .
\end{aligned}
$$

The definition of competitive equilibrium is standard.

Definition 1. A competitive equilibrium is an attainable allocation $\left(\hat{a}^{0}, \hat{\mu}^{0} ;\left(\hat{a}^{i}, \hat{c}^{i}\right.\right.$, $\left.\left.\hat{\mu}^{i}\right)_{i=1}^{N}\right)$ and nonnegative prices $(\hat{p}, \hat{q})$ such that:

(1) $\left(\hat{a}^{0}, \hat{\mu}^{0}\right)$ maximizes (1) subject to (2); and

(2) $\left(\hat{a}^{i}, \hat{c}^{i}, \hat{\mu}^{i}\right)$ maximizes (3) subject to (4) for each $i=1, \ldots, N$.

A competitive equilibrium links the initial endowment distribution, the speculation that arises from heterogeneous beliefs, and asset prices. In the following sections, we establish that an equilibrium exists and analyze its properties.

\section{SeCurity Design}

We first analyze the revenue maximization problem of the issuer. The issuer chooses optimally the set of securities taking as given security prices. Formally, the issuer with a unit of the risky asset chooses $\mu^{0} \in \mathcal{M}(\Sigma)$ to maximize the revenue

$$
\int_{\Sigma} q(\phi) d \mu^{0}
$$

\footnotetext{
${ }^{5}$ We denote safe asset holdings by $c^{i}$ since it delivers a unit of consumption in every state.
} 
subject to the feasibility constraint

$$
\int_{\Sigma} \phi(s) d \mu^{0} \leq s \text { for all } s .
$$

We call the above optimization problem the issuer's problem.

Lemma 1, in Appendix A, characterizes the equilibrium security price function in terms of an endogenous return vector $v=\left(v^{1}, \ldots, v^{N}\right) \in[1, \infty)^{N}$. The $i$ th component of the return vector $v^{i}$ plays the role of the Lagrangian multiplier on the budget constraint of Trader $i$ for prices $p$ and $q$. It reflects the marginal return of slackening the budget constraint. From the next section onward, the equilibrium determines the return vector. In the analysis of this section, we take it as given.

The lemma shows that the equilibrium security price function can be written as

$$
\hat{q}(\phi)=\max _{i} \frac{1}{\hat{v}^{i}} \int_{S} \phi(s) d F^{i}(s)
$$

for each security $\phi$ that is produced, where $\hat{v}$ is the equilibrium return vector. Intuitively, if $\phi$ is produced, then some trader, say Trader $i$, purchases it. Equation (10) sets her marginal utility from an extra unit of $\phi$ equal to her expected rate of return, $\hat{v}^{i}$. If equation (10) holds for every security, and not just the ones that trade, then we call $\hat{q}$ a canonical security price function for $\hat{v}^{6}$

Theorem 1 relates the maximum revenue to beliefs.

THEOREM 1. When $q$ is a canonical security price function for a given return vector $v, a$ solution to the issuer's problem exists and obtains revenue equal to

$$
r(v)=\int_{S} \max _{k}\left(v^{k}\right)^{-1} \widetilde{F}^{k}(x) d x .
$$

A formal proof is provided in Appendix A.2. An outline of the main arguments follows.

For an interval $(a, b)$, a security that pays zero for $s<a$, has a slope of 1 on $(a, b)$, and pays $b-a$ for $s>b$ is known to practitioners as a tranche; three are depicted in Figure 1B. The bounds are called attachment points. Trader $i$ values a tranche with attachment points $a<b$ as $\int_{a}^{b} \widetilde{F}^{i}(x) d x$, and since she has an opportunity cost of $v^{i}$ per unit of wealth, her willingness to pay for it is $\left(v^{i}\right)^{-1} \int_{a}^{b} \widetilde{F}^{i}(x) d x$.

Consider a candidate allocation of tranches. Suppose that two tranches in that allocation meet at $s$, the more senior is purchased by Trader 1 , and the more junior by Trader 2. The willingness to pay of Trader 1 for changing the attachment point to $s+\epsilon$ is approximately $\epsilon\left(v^{1}\right)^{-1} \widetilde{F}^{1}(s)$, and the willingness to pay of Trader 2 for the same change is $-\epsilon\left(v^{2}\right)^{-1} \widetilde{F}^{2}(s)$. Hence, the net effect on revenue of this change is the difference of the two, $\epsilon\left[\left(v^{1}\right)^{-1} \widetilde{F}^{1}(s)-\left(v^{2}\right)^{-1} \widetilde{F}^{2}(s)\right]$, and the issuer should increase (decrease) $s$ whenever this expression is positive (negative) until it equals zero.

\footnotetext{
${ }^{6}$ Any equilibrium remains so when replacing the security price function with the canonical one.
} 


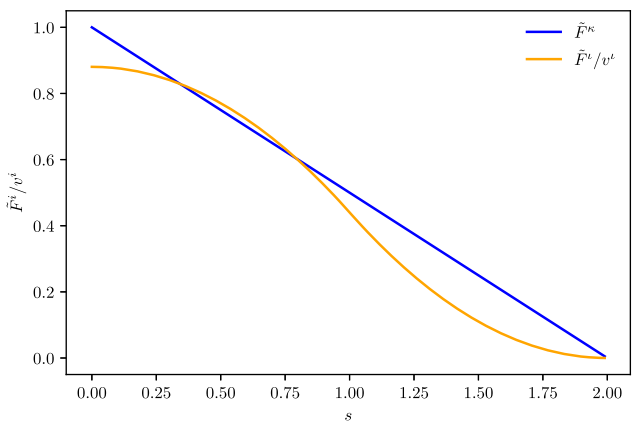

(A) Complementary CDF, before and after transformations

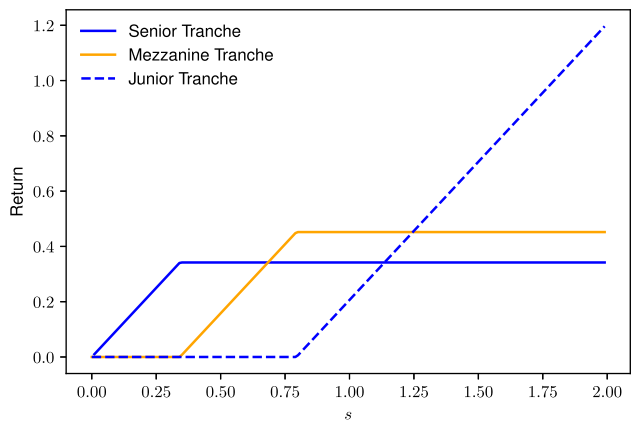

(B) Returns of equilibrium securities

FIGURE 1. Illustration of the solution to the issuer's problem.

Based on this, we can determine the revenue-maximizing allocation of tranches. To do so graphically, we plot the complementary CDF of Trader $i$ weighted by $\left(v^{i}\right)^{-1}$, that is, $\left(v^{i}\right)^{-1} \widetilde{F}^{i}(x)$, for each $i$ (illustrated in Figure 1A). ${ }^{7}$ As noted above, optimality requires that the attachment points occur where the lines cross. Hence, we create a tranche to be allocated to Trader $i$ (illustrated in Figure 1B) for each interval where $\left(v^{i}\right)^{-1} \widetilde{F}^{i}(x)$ exceeds all others. The revenue from this allocation of tranches is $r(v)$, which corresponds to the area under the upper envelope of these plots. We complete the proof by establishing that $r(v)$ is an upper bound on the revenue of the issuer for any increasing and feasible securities, not just tranches.

This graphical procedure explicitly links the revenue and securities to beliefs and disagreement. Each of the plots represents a trader's beliefs, adjusted for her required return. Increasing disagreement corresponds to increasing the gap between the weighted complementary CDFs. Thus, disagreement is a driving force behind securitization with heterogeneous beliefs.

This procedure also reveals which traders hold the safest and riskiest securities. The senior tranche, the safest security, is held by the trader with the highest $\left(v^{i}\right)^{-1} \widetilde{F}^{i}(0)$. Similarly, the equity tranche, the riskiest security, is held by the trader with the highest $\left(v^{i}\right)^{-1} \widetilde{F}^{i}(x)$ in a neighborhood of $\bar{s}$. If, and only if, the maximal complementary CDF switches exactly once is a debt-equity split optimal.

\section{Equilibrium}

This section shows existence of a competitive equilibrium and illustrates some of its properties. Equilibrium allocation and design of securities with heterogeneous beliefs typically leads to tranching and sorting (Corollary 1 ). As a consequence, equilibrium pricing can lead to overpricing of the underlying asset (Corollary 2).

\footnotetext{
${ }^{7}$ In the figure, Trader $\kappa$ thinks $s \sim U[0,2]$, Trader $\iota$ thinks $s$ is the independent sum of two $U[0,1]$ random variables, $v^{\kappa}=1$, and $v^{\iota}>1$.
} 


\subsection{Existence}

Using the observations in the previous section, we can now establish the existence of a competitive equilibrium.

THEOREM 2. A competitive equilibrium exists. The equilibrium utility of each agent and the price of the risky asset are unique.

Our approach to proving existence reduces the problem to finding an equilibrium return vector $\hat{v}$ via a fixed-point argument. From the above analysis, this vector determines the prices of securities, which securities trade in equilibrium, and the issuer's per unit revenue $r(\hat{v})$ (which must in turn equal the price of the risky asset). We illustrate the main arguments.

For simplicity, set the total supply of the risky asset to unity and the number of traders to 2. Consider a candidate return vector $v$ and its corresponding to the canonical security price function $q(\phi)=\max _{i}\left(v^{i}\right)^{-1} \mathbb{E}^{i}(\phi){ }^{8}$ Then $v$ is an equilibrium return vector if we can find a vector of securities $\left(\phi_{1}, \phi_{2}\right)$ that solves the Issuer's problem for $q$ with the following three properties. First, each Trader $i$ maximizes utility by purchasing a single unit of $\phi_{i}$ and investing the remainder in the safe asset. Second, whenever $p e_{a}^{i}+e_{c}^{i} \leq \mathbb{E}^{i}\left(\phi_{i}\right)$, Trader $i$ holds no safe asset and

$$
v^{i}=\mathbb{E}^{i}\left(\phi_{i}\right)\left(p e_{a}^{i}+e_{c}^{i}\right)^{-1} \geq 1 .
$$

This ensures that purchasing one unit of $\phi_{i}$ is exactly affordable for Trader $i$. Third, whenever $p e_{a}^{i}+e_{c}^{i}>\mathbb{E}^{i}\left(\phi_{i}\right)$, we have $v^{i}=1$, and since $q\left(\phi_{i}\right)<p e_{a}^{i}+e_{c}^{i}$, Trader $i$ holds a positive amount of the safe asset. This ensures that for Trader $i$, investing a unit of wealth in $\phi_{i}$ yields the same expected return as a unit of the safe asset.

The proof's main technical contribution develops a method for showing the existence of such a return vector. We define a correspondence with fixed points satisfying the above equilibrium conditions. The main difficulty is that standard fixed-point theorems do not apply as the correspondence may not be convex-valued. Since at least one trader holds the safe asset, $v^{i}=1$ for some $i$. However, there may be vectors $v^{*}$ and $v^{* *}$ satisfying this property while convex combinations do not, for instance when $v^{* 1}>1$ and $v^{* * 2}>1$. We develop a novel fixed-point theorem, based on the approximate selection theorem, that establishes existence of an equilibrium return vector. All details are in the Appendix.

\subsection{Equilibrium properties}

We now analyze some properties of equilibrium securitization. The following condition on beliefs will be useful and nests a number of common assumptions in the literature.

\footnotetext{
${ }^{8} \mathrm{~A}$ security $\phi$ that does not trade may not have uniquely determined prices. In equilibrium, we know from Lemma 1 that $q(\phi) \geq \max _{k}\left(v^{k}\right)^{-1} \mathbb{E}^{k}[\phi]$, but $q(\phi)$ cannot exceed $\max _{k}\left(v^{k}\right)^{-1} \mathbb{E}^{k}[\phi]$ by "too much" or the issuer would want sell $\phi$ (requiring that $q(\phi)=\max _{k}\left(v^{k}\right)^{-1} \mathbb{E}^{k}[\phi]$ ).
} 
Assumption 1 (Finite crossing). Each complementary CDF $\widetilde{F}^{i}$ is strictly decreasing on $(0, s)$, and for any distinct Traders $i, j$, and any $k>0$, there are finitely many points $x \in$ $[0, \bar{s}]$ for which $\widetilde{F}^{i}(x)=k \widetilde{F}^{j}(x)$.

Finite crossing is satisfied for the beliefs depicted in Figure 1. It guarantees that the inverse CDFs cross at most a finite number of times for any return vector. Since $\widetilde{F}^{i}$ is strictly decreasing, each trader's beliefs have full support. The remainder of the assumption is implied by the finite crossing of hazard rates, by the (strict) monotone likelihood ratio property, by A2 of Simsek (2013a), or by all of the complementary CDFs being smooth on $(0, \bar{s})$ and distinct.

Corollary 1. Under Assumption 1, each trader's equilibrium consumption is unique in every state, and an equilibrium exists where each security sold is a tranche, the intervals on which each tranche increases are disjoint, and each tranche is purchased by exactly one trader.

The corollary shows that under finite crossing, consumption is unique and there is an equilibrium with two salient properties. First, each security sold is a tranche. Second, each tranche is purchased only by a single trader. That is, the tranches sort the traders according to their beliefs. Moreover, unless one trader's beliefs first order stochastically dominate all others', there is no equilibrium where no securities are issued.

We contrast this allocation with that arising from homogeneous beliefs with heterogeneous risk aversion. Consider the case where each Trader $i$ has a CARA utility index, $u^{i}(x)=-\exp \left(-\alpha^{i} x\right)$ with $\alpha^{i}>0$ and common beliefs. With sufficiently large endowments of the safe asset, one can verify that Trader $i$ purchasing $\beta^{i} \equiv \bar{\alpha}\left(\alpha^{i}\right)^{-1}$ units of the risky asset for $\bar{\alpha} \equiv\left[\sum_{k=1}^{N}\left(\alpha^{k}\right)^{-1}\right]^{-1}$ and no securities being issued is an equilibrium. ${ }^{9}$

Predictably, securitization can increase the price of the risky asset. The price is above every trader's willingness to pay. When finite crossing holds and traders have the same expectation for the return, the asset price necessarily exceeds the common expected return.

Corollary 2. If $\mathbb{E}^{i}[s]=m$ for $i=1, \ldots, N$, then in any equilibrium, $\hat{p} \geq m$, with strict inequality whenever Assumption 1 holds.

Equilibrium securitization allocates tranches to traders who value them most, and thus increases the price of the risky asset above every trader's expectation. A graphical intuition for the result can be gained by inspecting Figure 1. Recall that the risky asset's price is the area under the upper envelope of $\left(v^{\iota}\right)^{-1} \widetilde{F}^{\iota}$ and $\widetilde{F}^{\kappa}$. Since $\mathbb{E}^{\kappa}[s]=$ $\int_{S} \widetilde{F}^{\kappa}(x) d x=m$ is the area under $\widetilde{F}^{\kappa}$, the price strictly exceeds the expected return.

This result extends beyond the case of equal means. Even the most optimistic trader can think that the price of the risky asset is overvalued. A sufficient condition, in addition to Assumption 1, is that her endowment is large enough and her beliefs do not first-order dominate every other trader's.

\footnotetext{
${ }^{9}$ See Ellis et al. (2019) for details, as well as the general case of risk aversion.
} 
Similar results appear in Harrison and Kreps (1978) and Fostel and Geanakoplos (2012). The latter is closest to our setting. They show the price of the risky asset can exceed its maximum return in a two-state environment with Arrow securities backed only by the risky asset and a continuum of traders ranked by optimism. In Harrison and Kreps (1978), overpricing occurs because a trader expects to be able to resell the risky asset in future states where other traders overvalue it.

By studying security design in a general equilibrium framework, one can identify linkages between endowments, pricing, and the allocation of securities. For instance, it can be easily seen that, apart from knife-edge cases, the trader who holds the senior tranche also holds the safe asset. Intuitively, each trader's willingness to pay for a tranche is her expectation of the tranche's return times the inverse of her equilibrium rate of return. Since $\widetilde{F}^{i}(0)=1$ for every Trader $i$, Trader $j$ has the highest willingness to pay for the senior tranche only if $\hat{v}^{j}=1$. That is, the trader who buys the senior tranche is indifferent between buying it and holding the safe asset. Consequently, the equilibrium entails an endogenous concentration of safe assets, even though all traders are risk-neutral.

In more specialized environments, these linkages can lead to more interpretable comparative statics. For instance, Theorem 4 of Simsek (2013a) relies on a general equilibrium effect through an assumption on the endowment distribution. In a dynamic setting, these links are central to understanding speculation and financial fluctuations, as studied by Caballero and Simsek (2019) and Martin and Papadimitriou (2019). General equilibrium effects would thus be essential for understanding financial stability with dynamics and securitization. While a full derivation is left to future research, our results lay the theoretical basis for a dynamic model.

\section{Pooling}

We have so far focused on the securitization of a single risky asset. In practice, issuers select a group of assets, pool them together, and then securitize the resulting pool. The standard rationale for this activity is diversification: while the pool is subject to aggregate risk, the idiosyncratic risks affecting individual assets are mitigated. We focus on an orthogonal rationale, namely that pooling may increase disagreement, and so create additional channels for screening beliefs via tranches targeted at particular traders.

In this section, we extend our baseline model to allow the securitization of multiple risky assets. The issuer purchases assets, creates one or more pools, and then sells securities backed by either one of the pools or by one of the assets. Traders accurately perceive the composition of the pool, but may disagree about the distribution of the aggregate return of the pool, or the distribution of the returns of the individual assets, or both. We focus on an underexplored dimension of securitization: the selection of which assets to include in each pool. We note that if diversification is the only motivation for pooling, then the optimal pool includes all assets with idiosyncratic returns.

Disagreement about the correlation between assets in the pool implies disagreement about the return of the pool itself, even when traders agree about the returns of individual assets. ${ }^{10}$ We define correlation disagreement by decomposing beliefs about

\footnotetext{
${ }^{10}$ With some abuse of terminology, we sometimes use "correlation" in place of the more appropriate "joint distribution" of returns.
} 
the joint returns into their marginal distributions and a copula. ${ }^{11}$ Traders disagree about the correlation when they have different copulas. While copulas are a common tool in structural finance, to our knowledge this definition is new.

We show that when traders agree about the correlation between assets, pooling does not benefit the issuer. In contrast, when they agree about the marginal distribution of each asset but disagree about their correlation, both pooling and tranching occur in equilibrium. Intuitively, with correlation agreement, traders disagree less about the sum of the assets than the assets individually. Conversely, with correlation disagreement, they disagree more about the sum of the assets. Tranching the pool allows the issuer to exploit the increased disagreement to raise revenue, leading to higher prices for the component assets.

In addition to the ample experimental evidence showing varying degrees of correlation neglect, evidence suggests correlation disagreement among sophisticated financial institutions. As mentioned in the Introduction, Griffin et al. (2013) show disagreement among ratings agencies. Nickerson and Griffin (2017) argue that both S\&P and Moody's understate the correlation parameter by a factor of four on average, even postcrisis. Their Table 1 indicates that the two agencies have different distributions of default correlations over a given set of deals, again suggesting disagreement about correlation. It appears that ratings agencies base their models on rudimentary assumptions about correlation with which one can quibble. For instance, while S\&P's baseline model includes the assumption that "correlation is likely to remain constant over time" (S\&P, 2015, p. 18), constant default intensity, even conditional on observables, is unlikely to hold (Duffie et al. 2009). Valuations of tranches from the same pool of assets also indicate either model misspecification or heterogeneous beliefs. In Duffie (2008, Table 4), the inferred correlation parameter for each tranche of a fixed pool of assets varies dramatically across tranches.

\subsection{Model}

Consider an economy as in Section 2, but with $J$ risky assets, in which Trader $i$ is endowed with $e_{j}^{i} \geq 0$ units of asset $j$. Uncertainty consists of a set of states $S^{J}=[0, \bar{s}]^{J}$. Trader $i$ has beliefs about the state that can be described by a nonatomic joint CDF, $F^{i}: S^{J} \rightarrow[0,1]$, with $F^{i}\left(s_{1}, \ldots, s_{J}\right)$ denoting the joint probability that risky asset $j$ 's return is less than or equal to $s_{j}$ for every $j=1, \ldots, J$. We also assume that all $J$-order partial derivatives exist for each $F^{i}(\cdot)$, and write $F_{j}^{i}(\cdot)$ for Trader $i$ 's (marginal) CDF of asset $j$ 's return $s_{j}$.

The issuer can sell any monotone securities based on each risky asset or one of several pools of assets it constructs. We normalize the set of potential pools so that a unit of each pool belongs to the unit simplex

$$
\Pi=\left\{z \in \mathbb{R}_{+}^{J}: \sum_{j=1}^{J} z_{j}=1\right\} .
$$

\footnotetext{
${ }^{11} \mathrm{~A}$ copula is the unique mapping from the marginal distributions to the joint distribution; see below for a formal definition.
} 
The issuer can create as many units of as many pools as it would like. However, as we shall see, there is an equilibrium where the issuer creates no more than $J$ pools. Thus, to simplify exposition, we allow the issuer to create exactly $J$ pools, $\pi^{k} \in \Pi$ for $k=J+$ $1, \ldots, 2 J$, where the indices start at $J$ to avoid confusion with the individual assets. ${ }^{12}$ Pool $\pi^{k}$ has $\pi_{j}^{k}$ units of asset $j$, and the issuer backs the corresponding securities with $a_{k}^{0}$ units of the pool. Hence, the issuer purchases $a_{j}^{0}$ units of asset $j$ to back the securities based only on asset $j$ and $\pi_{j}^{k} a_{k}^{0}$ units to back the securities based on pool $k$. Its total demand for asset $j$ is then $a_{j}^{0}+\sum_{k=J+1}^{2 J} \pi_{j}^{k} a_{k}^{0}$.

The securities issued for each pool or each asset belong to $\Sigma$. We set the security price function to be $q: \Pi \cup\{1, \ldots, J\} \times \Sigma \rightarrow \mathbb{R}_{+}$where $q(\pi, \phi)$ indicates the price of a security $\phi$ based on a pool $\pi$, and $q(j, \phi)$ the price of $\phi$ based on asset $j$. We denote the securities issued based on asset $j$ by a positive measure $\mu_{j}^{0} \in \mathcal{M}(\Sigma)$ and those based on pool $k$ by $\mu_{k}^{0} \in \mathcal{M}(\Sigma)$. We require that $\left(\mu_{1}^{0}, \ldots, \mu_{2 J}^{0}\right)$ maximizes

$$
\sum_{j=1}^{J} \int_{\Sigma} q(\phi, j) d \mu_{j}^{0}+\sum_{k=J+1}^{2 J} \int_{\Sigma} q\left(\phi, \pi^{k}\right) d \mu_{k}^{0}-\sum_{j=1}^{J} p_{j}\left[a_{j}^{0}+\left(\sum_{k=J+1}^{2 J} \pi_{j}^{k} a_{k}^{0}\right)\right]
$$

subject to

$$
\begin{gathered}
\int_{\Sigma} \phi\left(s_{j}\right) d \mu_{j}^{0} \leq a_{j}^{0} s_{j} \quad \text { for all } s_{j} \in S \text { and } j \leq J \\
\int_{\Sigma} \phi\left(s \cdot \pi^{k}\right) d \mu_{k}^{0} \leq a_{k}^{0} \pi^{k} \cdot s \quad \text { for all } s \in S^{J} \text { and } k>J .
\end{gathered}
$$

We denote by $F^{i}\left(\cdot ; \pi^{k}\right)$ the CDF describing Trader $i$ 's belief about the distribution of $s$. $\pi^{k}$. As above, $\widetilde{F}^{i}\left(\cdot ; \pi^{k}\right)$ denotes its complementary CDF.

Following our earlier analysis, if the return vector is $v$, then the price of a security $\phi \in \Sigma$ based on a pool characterized by $\pi^{k}$ is set to

$$
q\left(\pi^{k}, \phi\right)=\int_{S} \max _{i}\left(v^{i}\right)^{-1} \widetilde{F}^{i}\left(\cdot ; \pi^{k}\right) d \phi(x) .
$$

To simplify the analysis, we assume throughout this section, and without further mention, that $e_{c}^{i}$ is sufficiently large for each $i$ so that each trader can afford to purchase as many units of any security as would be supplied in equilibrium and, therefore, that $\left(v^{i}\right)^{-1}=1$ for each $i$. All definitions are extensions from Section 2; see Appendix B for details and proofs. We call such an economy a pooling economy.

\subsection{Equilibrium pools}

First, we show that an equilibrium exists.

THeORem 3. A competitive equilibrium exists for any pooling economy.

\footnotetext{
${ }^{12}$ We index pools by superscript with subscripts indicating the amount of each asset in each pool.
} 
The main complication in the proof is that the revenue from optimally tranching a pool of assets is not a concave function of the pool's composition. As we shall see, combining two pools into a single pool may either increase or decrease the revenue from securitization. Allowing the issuer to form multiple pools in varying proportions convexifies its problem.

We are interested in when pooling occurs in equilibrium.

Definition 2. A pool is proper if it contains positive amounts of at least two assets. A proper pool circulates in an equilibrium if securities based on a proper pool are sold.

A proper pool is distinguished from a trivial "pool" containing only a single asset. We use it to distinguish whether or not pools are necessary for profit maximization. Pooling strictly increases profit if a proper pool circulates in every equilibrium, and does not when no proper pool circulates in any equilibrium.

Now, we turn to the properties of equilibria. First, we decompose each trader's beliefs over the assets' returns into the marginal CDF of each asset and a copula that aggregates them. A copula is a joint CDF on $[0,1]^{J}$ where each dimension has a uniform marginal distribution on [0, 1]. Formally, Sklar's theorem (2.10.9 of Nelson (2006)) guarantees that for each Trader $i$, there is a (unique) copula $C^{i}:[0,1]^{J} \rightarrow[0,1]$ so that

$$
F^{i}\left(s_{1}, \ldots, s_{J}\right)=C^{i}\left(F_{1}^{i}\left(s_{1}\right), \ldots, F_{J}^{i}\left(s_{J}\right)\right) .
$$

We will define correlation agreement and disagreement in terms of the copula.

Copulas are a common tool in econometrics and structural finance. Econometrically, they allow for separate estimation of the marginal distribution and joint distribution. If, as is common, the copula is known or assumed to belong to a parametric family, then that parameter can be estimated and interpreted. Pricing derived securities is then done via the estimated copula.

5.2.1 Correlation agreement We say that traders agree on the correlation if they have the same copula: $C^{i}=C$ for all $i=1, \ldots, N$. In this case, they aggregate the marginal distributions in the same way. Traders must disagree on the correlation for pooling to play a role in our model.

We also say that there is undominated disagreement about asset $j$ if the traders' beliefs about $s_{j}$ satisfy Assumption 1, and no trader's beliefs about that asset first-order dominate every other's. In this case, no trader is more optimistic about asset $j$ than all the others, and the traders' marginal CDFs are not too similar. Undominated disagreement rules out inessential pooling of assets. For instance, if Trader $i$ has beliefs about assets $j$ and $k$ that first-order dominate all the others, then she may purchase all the securities backed by a proper pool containing the two assets.

THEOREM 4. If traders agree on the correlation, then there is an equilibrium where no proper pool circulates. Moreover, if the common copula admits a strictly positive density and there is undominated disagreement about asset $j$, then there is no equilibrium where a proper pool containing asset $j$ circulates. 
When traders disagree about individual assets but agree on the correlation, pooling assets together causes them to disagree less. We illustrate this with an extreme case where pooling leads to agreement, even though traders disagree about individual assets. Suppose that Traders 1 and 2 agree that assets 1 and 2 are independent, and that $F_{1}^{1}(x)=F_{2}^{2}(x)=F(x)$ and $F_{2}^{1}(x)=F_{1}^{2}(x)=G(x)$. That is, the traders agree that one asset has marginal distribution $F$ and the other $G$, but disagree as to which. Then they agree about the distribution of a pool with an equal share of each asset-specifically, $F^{i}\left(x ;\left(\frac{1}{2}, \frac{1}{2}\right)\right)=\int_{0}^{\bar{s}} F(2 x-y) g(y) d y$ for $i=1,2$ where $g$ is the density of $G$. Hence, the revenue of securitizing the pool is $\frac{1}{2} \mathbb{E}^{1}\left[s_{1}+s_{2}\right]=\frac{1}{2} \mathbb{E}^{2}\left[s_{1}+s_{2}\right]$. By Corollary 2 , the sum of the revenues from securitizing each asset individually strictly exceeds this whenever $F$ and $G$ satisfy Assumption 1. The above theorem extends the logic behind this observation to every pool, all marginal distributions, and every common copula.

5.2.2 Correlation disagreement We now turn to the pooling decision when traders disagree about correlation. We restrict attention to the special case where traders have the same marginal beliefs about each asset's return. However, traders may disagree on the correlation, that is, have different copulas. We show that pooling and tranching allows the issuer to exploit the disagreement to increase its revenue.

We say that traders agree on the marginals if there exists CDFs $\left(F_{1}, \ldots, F_{J}\right)$ so that each trader believes that the marginal return of asset $j$ is described by $F_{j}$. Under this assumption, if securities can only be backed by a single asset, then every trader agrees on the return of each security, and the issuer cannot increase its revenue beyond the expected value of assets themselves. However, if at least two traders have differing copulas, pooling occurs in equilibrium.

THEOREM 5. If traders agree on the marginals, then there is an equilibrium where at least one proper pool circulate. Moreover, if there exist assets $j, j^{\prime}$, and Traders $i, i^{\prime}$ so that $\mathbb{E}^{i}\left[s_{j} \mid s_{j^{\prime}}=x\right] \neq \mathbb{E}^{i^{\prime}}\left[s_{j} \mid s_{j^{\prime}}=x\right]$ for some $x \in(0, \bar{s})$, then a proper pool circulates in every equilibrium.

When traders agree about individual assets but disagree on their correlation, then pooling assets together causes them to disagree more. To illustrate, suppose Trader 1 thinks that the two assets are perfectly correlated while Trader 2 thinks they are independent, but that they agree that both assets have return distributed uniformly on $[0,1]$. Since traders agree on the marginal distributions, they also agree on the value of any security backed by a single asset. Hence, the maximal revenue from tranching an individual asset is its expectation. Similarly, they agree on the expected value of an entire pool. However, they may disagree on the probability that the pool yields an extreme return. For instance, Trader 1 thinks that the value of a senior tranche of the pool $\left(\frac{1}{2}, \frac{1}{2}\right)$ with attachment point $y \leq \frac{1}{2}$ is $y$ while Trader 2 thinks it is $2 y^{2}$.

Pooling causes an increase in disagreement whenever the copulas are sufficiently different. A sufficient condition is given in the above theorem: $\mathbb{E}^{i}\left[s_{j} \mid s_{j^{\prime}}=x\right] \neq \mathbb{E}^{i^{\prime}}\left[s_{j} \mid s_{j^{\prime}}=\right.$ $x$ ]. Note that this condition is necessary for Traders $i$ and $i^{\prime}$ to disagree on the correlation 
coefficient between $s_{j}$ and $s_{j^{\prime}}$, since $\mathbb{E}^{i}\left[s_{j} s_{j^{\prime}}\right]=\mathbb{E}^{i}\left[\mathbb{E}^{i}\left[s_{j} \mid s_{j^{\prime}}\right] s_{j^{\prime}}\right]$. Intuitively, Trader $i$ 's conditional expectation about asset $j$ 's return is different from that of Trader $i^{\prime}$. This weak condition suffices for pooling to increase disagreement strictly.

We can also characterize the equilibrium pools and tie them to the endowments in more specialized examples. For instance, consider an economy with two assets and two traders. Suppose that Trader $i$ believes that the correlation between the two risky assets is $\rho_{i}$, but both think that each asset has the same marginal distribution. In particular, Trader $i$ believes the risky assets' returns are independent with probability $\rho_{i}$ and otherwise perfectly correlated. ${ }^{13}$ Each asset's marginal density is $f$, where $f$ is differentiable, log-concave, and symmetric about its mean $m=\int_{S} x f(x) d x .^{14}$

There is a unique equilibrium with a single pool in this example. ${ }^{15}$ All risky assets are pooled: the equilibrium pool that circulates is $\pi^{*}$ for $\pi_{1}^{*}=\frac{\sum_{i=1}^{N} e_{1}^{i}}{\sum_{i=1}^{N} e_{1}^{i}+\sum_{i=1}^{N} e_{2}^{i}}$. Letting $R(\pi)$ be the revenue from optimally securitizing a pool $\pi \in \Pi$, the prices of the risky assets are

$$
\begin{aligned}
& \hat{p}_{1}=R\left(\pi^{*}\right)+\pi_{2}^{*}\left[\frac{\partial}{\partial \pi_{1}} R\left(\pi^{*}\right)-\frac{\partial}{\partial \pi_{2}} R\left(\pi^{*}\right)\right] \\
& \hat{p}_{2}=R\left(\pi^{*}\right)-\pi_{1}^{*}\left[\frac{\partial}{\partial \pi_{1}} R\left(\pi^{*}\right)-\frac{\partial}{\partial \pi_{2}} R\left(\pi^{*}\right)\right] .
\end{aligned}
$$

The revenue $R$ is strictly concave and inverse- $\mathrm{U}$ shaped in $\pi_{1}$, and $\hat{p}_{j} \geq m$ for each $j .{ }^{16}$ The optimal securitization is a junior tranche with attachment point at the mean and a senior tranche that pays the remainder.

5.2.3 Wrap up With general belief disagreement and two assets, incentives to create pools depend on the revenue from optimally securitizing a pool $\pi \in \Pi$ :

$$
\begin{aligned}
R(\pi) & =\int_{S}\left\{\max _{i} \widetilde{F}^{i}(x ; \pi)\right\} d x \\
& =\int_{S}\left\{\max _{i}\left[1-\int_{S} \partial_{2} C^{i}\left(F_{1}^{i}\left(\left(\pi_{1}\right)^{-1}\left(x-\pi_{2} y_{2}\right)\right), F_{2}^{i}\left(y_{2}\right)\right) f_{2}^{i}\left(y_{2}\right) d y\right]\right\} d x .
\end{aligned}
$$

A proper pool circulates in equilibrium whenever $R(\pi)>\pi_{1} R((1,0))+\pi_{2} R((0,1))$ for some proper $\pi .{ }^{17}$ Similarly, no proper pool circulates when $R(\pi)<\pi_{1} R((1,0))+$ $\pi_{2} R((0,1))$ for every proper $\pi$. When $R$ is strictly concave, as in the example above, then there exists an equilibrium with a single pool containing all assets (regardless of the number of assets).

Theorems 4 and 5 provide sharp and easily interpretable conditions for the inequalities to hold. Pooling in the general case depends on the extent of both marginal belief

\footnotetext{
${ }^{13}$ The copula is $C^{i}(x, y)=\rho_{i} x y+\left(1-\rho_{i}\right) \min \{x, y\}$.

${ }^{14}$ The uniform, normal, logistic, and truncated normal distributions, among others, are log-concave and symmetric.

${ }^{15}$ In fact, all equilibria are essentially equivalent to this one. There may be multiple pools but each has the same fraction of each of the assets.

${ }^{16}$ See Ellis et al. (2019) for details.

${ }^{17}$ See the proof of Theorems 4 and 5 for the formula for more than two assets.
} 
disagreement and correlation disagreement. A complete characterization is left for future research.

\section{Appendix A: Details from Proofs from Sections 3 and 4}

\section{A.1 Expository lemmas}

We begin by offering two expository lemmas. Lemma 1 establishes what prices must look like in any equilibrium, motivating our definition of $q$ in the issuer's problem below. Lemma 1 establishes that the revenue in the issuer's problem must equal the price of the risky asset in equilibrium.

Lemma 1. For any equilibrium allocation $\left(\hat{a}^{0}, \hat{\mu}^{0} ;\left(\hat{a}^{i}, \hat{c}^{i}, \hat{\mu}^{i}\right)_{i=1}^{N}\right)$ and prices $(\hat{p}, \hat{q})$,

$$
\hat{q}(\phi) \geq \max _{k}\left(\hat{v}^{k}\right)^{-1} \mathbb{E}^{k}[\phi]
$$

for all $\phi \in \Sigma$, with equality $\hat{\mu}^{0}$-a.e., where

$$
\hat{v}^{k}=\max \left\{1, \frac{\int\left[\int_{\Sigma} \phi(s) d \hat{\mu}^{k}\right] d F^{k}}{\int_{\Sigma} \hat{q} d \hat{\mu}^{k}}\right\}
$$

is the equilibrium return vector. Furthermore, for every Trader $i \hat{q}(\phi)=\left(\hat{v}^{i}\right)^{-1} \mathbb{E}^{i}[\phi]$ for $\hat{\mu}^{i}$-a.e. $\phi \in \Sigma$.

Proof. Let ( $\hat{p}, \hat{q})$ be equilibrium prices, and suppose for contradiction that there exists a security $\phi^{*}$ such that $\hat{q}\left(\phi^{*}\right)<\left(\hat{v}^{k}\right)^{-1} \mathbb{E}^{k}[\phi]$ for some Trader $k$. Consider the following alternative portfolios. If $\hat{c}^{k}>0$, then consider the allocation $c=\hat{c}^{k}-q\left(\phi^{*}\right) \epsilon$ and $\mu=$ $\hat{\mu}^{k}+\epsilon \delta_{\left\{\phi^{*}\right\}}$. Then her utility changes by

$$
\epsilon \int_{S}\left(\phi^{*}(s)-q\left(\phi^{*}\right)\right) d F^{k}>0,
$$

contradicting utility maximization. Similarly, if $\hat{c}_{k}=0$, then $\int \hat{q} d \hat{\mu}^{i}>0$ and let $w=$ $\int \phi d \hat{\mu}^{k}$. Consider $\mu=\hat{\mu}^{k}\left(1-\frac{q\left(\phi^{*}\right)}{\int \hat{q} d \hat{\mu}^{k}} \epsilon\right)+\epsilon \delta_{\left\{\phi^{*}\right\}}$ and $c=0$. Then her utility from $\mu$ differs from her utility from $\hat{\mu}^{k}$ by

$$
\epsilon \int_{S}\left(\phi^{*}(s)-w(s) \frac{\hat{q}\left(\phi^{*}\right)}{\int \hat{q} d \hat{\mu}^{k}}\right) d F^{k} \geq \epsilon \int_{S}\left(\phi^{*}(s)-\left(\hat{v}^{k}\right) \hat{q}(\phi)\right) d F^{k}>0,
$$

again contradicting utility maximization.

Now, define $B=\left\{\phi \in \Sigma: \hat{q}(\phi)>\max _{k}\left(\hat{v}^{k}\right)^{-1} \int \phi(s) d F^{k}\right\}$, and noting $B$ is Borel, suppose that $\hat{\mu}^{0}(B)>0$. Since $\hat{q}(\phi)>0$ for all $\phi \in B$, attainability requires that there exists a Trader $i$ such that $\hat{\mu}^{i}(B)>0$. But then reducing $\hat{\mu}^{i}(B)$ and increasing $c^{i}$ is affordable and increases utility by the same arguments as above. This observation concludes the proof. 
Remark 3. One consequence of the lemma is that $\hat{c}^{i}>0$ only if $\hat{v}^{i}=1$. Intuitively, $\hat{v}^{i}$ is the highest marginal utility per unit of wealth, in terms of the safe asset (the numeraire), that can be obtained from any security. If $\hat{v}^{i}>1$, then some security offers a better marginal return than the safe asset.

Lemma 2. In any equilibrium allocation $\left(\hat{a}^{0}, \hat{\mu}^{0} ;\left(\hat{a}^{i}, \hat{c}^{i}, \hat{\mu}^{i}\right)_{i=1}^{N}\right)$ with prices $(\hat{p}, \hat{q})$, equilibrium return vector $\hat{v}, \hat{p}$ equals the maximum revenue to the issuer per unit securitized and the issuer's profits are equal to zero.

Proof. Let $\hat{r}$ be the maximal revenue per unit of risky asset given equilibrium security prices $\hat{q}$. Then profit equals $(\hat{r}-\hat{p}) \hat{a}^{0}$ since selling securities that obtain less than $\hat{r}$ contradicts profit maximization. If $\hat{p}<\hat{r}$, then no maximum profit exists. If $\hat{p}>\hat{r}$, then $\hat{a}^{0}=0$. By Lemma $1, \hat{r} \geq \frac{1}{\hat{v}^{i}} \mathbb{E}^{i}[s]$ for all $i$, so $\hat{a}^{i}=0$. Then traders sell their endowment of the risky asset and hold only the safe asset, a contradiction to feasibility of the allocation. Conclude $\hat{p}=\hat{r}$ and so profit is zero.

\section{A.2 Proof of Theorem 1}

Throughout, we let $\lambda$ be the Lebesgue measure restricted to $S$.

Lemma 3. Given a return vector $v$, suppose that $q(\phi)=\max _{i}\left(v^{i}\right)^{-1} \mathbb{E}^{i}[\phi]$ for any $\phi \in \mathcal{B}$. For any $\mu \in \mathcal{M}(\mathcal{B})$, there are $\left(\psi_{1}, \ldots, \psi_{N}\right) \in \Sigma^{N}$ such that each $\psi_{i}$ is right continuous and $\sum_{i=1}^{N} \psi_{i}(s) \leq s$ such that $\sum q\left(\psi_{i}\right)=\int q d \mu$.

Proof. Fix any $\mu \in \mathcal{M}(\Sigma)$. Then the set

$$
B_{i}(v)=\left\{\phi \in \Sigma: q(\phi)=\left(v^{i}\right)^{-1} \mathbb{E}^{i}[\phi(s)] \text { and } k \geq i \text { whenever } q(\phi)=\left(v^{k}\right)^{-1} \mathbb{E}^{k}[\phi(s)]\right\}
$$

is a Borel set and the collection $\left\{B_{i}(v)\right\}_{i=1}^{N}$ is a partition of $\Sigma$. Define the securities $\psi_{i}$, $i=1, \ldots, N$, as

$$
\psi_{i}(s)=\inf _{x>s} \int_{B_{i}(v)} \phi(s) d \mu
$$

Since securities are nondecreasing, $\psi_{i}$ is right continuous and

$$
\psi_{i}(s)=\int_{B_{i}(v)} \phi(s) d \mu
$$

almost surely. By Fubini's theorem and the definition of $q$, we have for each $i$ that

$$
q\left(\psi_{i}\right)=\max _{k}\left(v^{k}\right)^{-1} \mathbb{E}^{k}\left[\psi_{i}(s)\right]=\left(v^{i}\right)^{-1} \mathbb{E}^{i}\left[\psi_{i}(s)\right]=\int_{B_{i}(v)} q(\phi) d \mu .
$$

It follows from the definition of $\left\{B_{i}(v)\right\}_{i=1}^{N}$ that $\int q(\phi) d \mu^{\prime}=\sum_{i=1}^{N} q\left(\psi_{i}\right)=\int q(\phi) d \mu$. 
Thus, in the issuer's problem for $v$ it is without loss to restrict the choice to vectors of securities $\left(\phi_{1}, \ldots, \phi_{N}\right)$ that are right continuous and satisfy $\sum_{i=1}^{N} \phi_{i}(s) \leq s$. Let $\gamma_{i}$ be the Lebsegue-Stieltjes measure for $\phi_{i}$, that is, the Borel measure so that for every half-open interval $(x, y], \gamma_{i}((x, y])=\phi_{i}(y)-\phi_{i}(x)$. Observe $\psi_{i}(s)=\gamma_{i}([0, s])=\int_{S} \chi_{[x, \bar{s}]}(s) \gamma_{i}(d x)$ for all $s$. Then, for any $i, j \in\{1, \ldots, N\}$,

$$
\mathbb{E}^{i}[\phi]=\mathbb{E}^{i}\left[\int_{S} \chi_{[x, \bar{s}]} d \gamma_{j}\right]=\int_{S} \mathbb{E}^{i}\left[\chi_{[x, \bar{s}]}\right] d \gamma_{j}=\int_{S}\left(1-F^{i}(x)\right) d \gamma_{j}
$$

Therefore, we can restate the issuer's problem as the choice of measures $\left(\gamma_{1}, \ldots, \gamma_{N}\right)$ to maximize

$$
\begin{gathered}
\sum_{i=1}^{N}\left(v^{i}\right)^{-1} \int_{S}\left(1-F^{i}(x)\right) \gamma_{i}(d x) \\
\text { s.t. } \sum_{i=1}^{N} \gamma_{i}([0, s]) \leq s \text { for all } s
\end{gathered}
$$

For each $i=1, \ldots, N$, define the sets

$$
M_{i}(v)=\left\{x \in[0, \bar{s}]:\left(v^{i}\right)^{-1}\left[1-F^{i}(x)\right]=\max _{k}\left(v^{k}\right)^{-1}\left[1-F^{k}(x)\right]\right\}
$$

and

$$
M_{i}^{*}=M_{i}(v) \backslash\left[\bigcup_{j<i} M_{j}(v)\right]
$$

and consider the measures $\gamma_{i}^{*}$ such that $\gamma_{i}^{*}(B)=\lambda\left(B \cap M_{i}^{*}\right)$ for all Borel sets $B$.

Let $G^{*}(x)=\max _{i}\left(v^{i}\right)^{-1} \widetilde{F}^{i}(x)$. Then the issuer's revenue for a vector of securities $\left(\psi_{1}^{*}, \ldots, \psi_{N}^{*}\right)$ where each $\psi_{i}^{*}$ is derived from $\gamma_{i}^{*}$ is

$$
\sum_{i=1}^{N} \int_{M_{i}^{*}} G^{*}(x) \gamma_{i}^{*}(d x)=\int_{S} G^{*}(x) d x=r(v)
$$

For any feasible measures $\left(\gamma_{1}, \ldots, \gamma_{N}\right)$, we have

$$
\begin{aligned}
\sum_{i=1}^{N}\left(v^{i}\right)^{-1} \int_{S}\left(1-F^{i}(x)\right) \gamma_{i}(d x) & =\sum_{i=1}^{N} \sum_{k=1}^{N}\left(v^{i}\right)^{-1} \int_{M_{k}^{*}}\left(1-F^{i}(x)\right) \gamma_{i}(d x) \\
& \leq \sum_{i=1}^{N} \int_{S} G^{*}(x) \gamma_{i}(d x)=\int_{S} G^{*}(x)\left[\sum_{i=1}^{N} \gamma_{i}\right](d x) \\
& \leq \int_{S} G^{*}(x) d x
\end{aligned}
$$


where the last inequality follows from $\sum_{i=1}^{N} \gamma_{i}$ first-order stochastically dominating $\lambda$ and $G^{*}(x)$ decreasing. ${ }^{18}$ Hence, the securities $\left(\psi_{1}^{*}, \ldots, \psi_{N}^{*}\right)$ maximize revenue and obtain $r(v)$, completing the proof.

\section{A.3 Proof of Theorem 2}

We know from Theorem 1 that the constraint (2) binds and it is without loss to consider only $N$ securities. Let

$$
\Sigma^{*}=\left\{\left(\phi_{1}, \ldots, \phi_{N}\right) \in \Sigma^{N}: \sum_{i=1}^{N} \phi_{i}(s)=s \text { for any } s \in[0, \bar{s}]\right\}
$$

be the set of securities that the issuer may choose. Observe that if $\left(\phi_{1}, \ldots, \phi_{N}\right) \in \Sigma^{*}$, then each $\phi_{i}$ has the property that $\left|\phi_{i}(s)-\phi_{i}\left(s^{\prime}\right)\right| \leq\left|s-s^{\prime}\right|$ for all $s, s^{\prime}$. Also, the set of vectors $\left(\phi_{1}, \ldots, \phi_{N}\right)$ such that $\sum \phi_{i}(s)=s$ for all $s$ is closed in the supnorm. Hence, $\Sigma^{*}$ is a compact set by Arzelà's theorem.

Lemma 4. In any equilibrium, $\hat{p}=r(\hat{v})$ and the issuer's profits are equal to zero.

Proof. That profits are equal to zero is standard. Let $\hat{r}$ be the maximal revenue per unit of asset given equilibrium security prices $\hat{q}$. Then $\hat{p} \geq \hat{r}$. By Lemma $1, \hat{q}(\phi) \geq$ $\max _{k}\left(\hat{v}^{k}\right)^{-1} \mathbb{E}^{k}[\phi]$, and thus $\hat{r} \geq r(\hat{v})$ by Theorem 1. Since $\hat{q}(\phi)=\max _{k}\left(\hat{v}^{k}\right)^{-1} \mathbb{E}^{k}[\phi] \hat{\mu}_{0^{-}}$ a.e.,

$$
\hat{a}_{0} \hat{r}=\int_{\mathcal{B}} \max _{i}\left(v^{i}\right)^{-1} \mathbb{E}^{i}[\phi] \hat{\mu}_{0}(d \phi) \leq \hat{a}_{0} r(\hat{v}) .
$$

We conclude that $\hat{r}=r(\hat{v})$ if $\hat{a}_{0}>0$. Now if $\hat{p}>r(\hat{v}), \hat{p}>\left(\hat{v}^{k}\right)^{-1} \mathbb{E}^{k}[s]$ for any $k$. An argument similar to the one in Lemma 1 establishes that no trader will demand the asset. Since by the previous argument $\hat{a}_{0}>0$ implies that $\hat{p}>\hat{r}, \hat{a}_{0}$ must also be equal to zero. A contradiction is then obtained by Walras law.

Define the aggregate endowment $e_{a}=\sum_{i=1}^{N} e_{a}^{i}$, and for any vector $v \in[1, \infty)^{N}$, let

$$
M_{i}(v)=\left\{x \in[0, \bar{s}]:\left(v^{i}\right)^{-1}\left[1-F^{i}(x)\right]=\max _{k}\left(v^{k}\right)^{-1}\left[1-F^{k}(x)\right]\right\}
$$

and let

$$
\mathcal{C}(v)=\left\{\left(\phi_{1}, \ldots, \phi_{N}\right) \in \Sigma^{*}: \int_{M_{i}(v)^{c}} 1 d \phi_{i}=0, \text { for all } i\right\}
$$

where $\int g d h$ represents the integral of $g$ with respect to the Lebesgue-Stieltjes measure for $h .{ }^{19}$ The set $\mathcal{C}(v)$ is clearly convex and is compact as a closed subset of $\Sigma^{*}$.

\footnotetext{
${ }^{18}$ Possibly after adding an atom at zero and normalizing to make both probability measures.

${ }^{19}$ For a set $E, E^{c}$ is its complement.
} 
Given a return vector $v$ and $\Phi=\left(\phi_{1}, \ldots, \phi_{N}\right) \in \Sigma^{*}$, define

$$
g_{i}(v, \Phi)=e_{a} \frac{\int\left[1-F^{i}(x)\right] d \phi_{i}}{e_{c}^{i}+r(v) e_{a}^{i}},
$$

where $r(v)$ is the issuer's revenue given $v$. Note there exists $\bar{v}$ such that $g_{i}(v, \Phi) \in[0, \bar{v}]$ for every $i$ and $\Phi \in \mathcal{C}(v) .{ }^{20}$ Define a function $G$ from $[1, \bar{v}]^{N}$ to $[0, \bar{v}]^{N}$ by

$$
G=(v, \Phi) \mapsto\left(g_{1}(v, \Phi), \ldots, g_{N}(v, \Phi)\right)
$$

and a function

$$
H=\left(v^{1}, \ldots, v^{N}\right) \mapsto\left(\max \left\{v^{1}, 1\right\}, \ldots, \max \left\{v^{N}, 1\right\}\right) .
$$

The function $H$ projects $[0, \bar{v}]^{N}$ onto $[1, \bar{v}]^{N}$.

Lemma 5. Any $\hat{v} \in[1, \bar{v}]^{N}$ and $\hat{\Phi} \in \mathcal{C}(\hat{v})$ such that $\hat{v}=H \circ G(\hat{v}, \hat{\Phi})$ defines an equilibrium.

Proof. Consider $\hat{v} \in[1, \bar{v}]^{N}$ and $\hat{\Phi} \in \mathcal{C}(\hat{v})$ such that $\hat{v}=H(g(\hat{v}, \hat{\Phi}))$. Let

$$
\hat{q}(\phi)=\max _{k}\left(\hat{v}^{k}\right)^{-1} \mathbb{E}^{k}[\phi]
$$

for all $\phi \in \mathcal{B}$ and

$$
\hat{p}=r(\hat{v})=\sum_{i=1}^{N}\left(\hat{v}^{i}\right)^{-1} \int_{S}\left(1-F^{i}(x)\right) d \hat{\phi}_{i} .
$$

For each $i$,

$$
\begin{aligned}
& \hat{v}^{i}=1>g_{i}(\hat{v}, \hat{\Phi}) \quad \Longrightarrow \quad e_{c}^{i}+\hat{p} e_{a}^{i}>e_{a} \int\left[1-F^{i}(x)\right] d \hat{\phi}_{i}=e_{a} \hat{q}\left(\hat{\phi}_{i}\right) \\
& \hat{v}^{i}=g_{i}(\hat{v}, \hat{\Phi}) \quad \Longrightarrow \quad e_{c}^{i}+\hat{p} e_{a}^{i}=e_{a}\left(\hat{v}^{i}\right)^{-1} \int\left[1-F^{i}(x)\right] d \hat{\phi}_{i}=e_{a} \hat{q}\left(\hat{\phi}_{i}\right) .
\end{aligned}
$$

We must have $\hat{v}^{i}=1$ for some $i$, or else

$$
\sum_{i=1}^{N}\left(e_{c}^{i}+\hat{p} e_{a}^{i}\right)=\sum_{i \in I} \hat{q}\left(\hat{\phi}_{i}\right) e_{a}=\hat{p} \sum_{i=1}^{N} e_{a}^{i},
$$

a contradiction. Thus, markets clear when $\hat{\mu}_{0}=\sum e_{a} \delta_{\hat{\phi}_{i}}, \hat{\mu}^{i}=e_{a} \delta_{\hat{\phi}_{i}}, \hat{a}_{0}=e_{a}, \hat{c}^{i}=e_{c}^{i}+$ $\hat{p} e_{a}^{i}-\hat{q}\left(\hat{\phi}_{i}\right)$ and $\hat{a}^{i}=0$, where $\delta_{x}$ is the Dirac measure on $\{x\}$. We now show these are also optimal for $\hat{q}(\cdot)$ and $\hat{p}$. By Theorem 1, the issuer's maximal revenue is $r(\hat{v})$, so it is optimal for the issuer to produce $e_{a}$ units each of securities $\phi_{i}$ using $e_{a}$ units of the asset.

${ }^{20}$ One such upper bound is $\max _{i}\left[\left(e^{c}+e_{a} \int_{0}^{\bar{s}}\left[1-F^{i}(x)\right] d x\right) /\left(e_{c}^{i}\right)\right]$. 
Now consider $a^{i}, c^{i}$, and a measure $\mu^{i}$ over $\Sigma$ that satisfy Trader $i$ 's budget constraint. Then

$$
\begin{aligned}
\mathbb{E}^{i}[s] a^{i}+\int_{\mathcal{B}} \mathbb{E}^{k}[\phi] d \mu^{i}(\phi)+c^{i} & \leq \hat{v}^{i} \hat{p} a^{i}+\hat{v}^{i} \int \hat{q}\left(\phi_{i}\right) d \mu^{i}(\phi)+c^{i} \\
& \leq \hat{v}^{i}\left(e_{c}^{i}+\hat{p} e_{a}^{i}\right)=\hat{c}^{i}+e_{a} \mathbb{E}^{i}\left[\hat{\phi}_{i}\right]
\end{aligned}
$$

and thus $\left(\hat{c}^{i}, \hat{a}^{i}, \hat{\mu}^{i}\right)$ maximizes utility given $\hat{p}$ and $\hat{q}(\cdot)$.

We now establish such a fixed point exists. Observe that the usual fixed-point theorems do not apply since the set $\{H \circ G(v, \Phi): \Phi \in \mathcal{C}(v)\}$ may not be convex. The following lemma is key.

Lemma 6. Consider compact, convex, nonempty subsets $X, Y$ of normed linear spaces and a correspondence $K: X \rightrightarrows X$. If there exist a convex, compact, and nonempty valued correspondence $\tilde{K}: X \rightrightarrows Y$ with a closed graph and a continuous function $H: X \times Y \rightarrow X$ such that $K(x)=\{H(x, y): y \in \tilde{K}(x)\}$ for each $x \in X$, then there exists $x^{*} \in X$ such that $x^{*} \in K\left(x^{*}\right)$.

Proof. Since the correspondence $\tilde{K}(\cdot)$ satisfies the assumptions of the approximate selection theorem (Theorem 6.5 of Shapiro (2016)), for any $m>0$, there exists a continuous function

$$
\gamma^{m}: X \rightarrow Y
$$

such that $\operatorname{graph}\left(\gamma^{m}\right) \subset \bigcup_{(x, y) \in \operatorname{graph}(G)} B_{1 / m}(x, y)$, where $B_{1 / m}(x, y)$ is an the open ball with diameter $1 / m$ centered on $(x, y)$. The function $h^{m}=x \mapsto H\left(x, \gamma^{m}(x)\right)$ is a continuous functions from $X$ to $X$. Then for each $m \in \mathbb{N}, h^{m}$ has a fixed point $x^{m}$ by Schauder's fixed-point theorem (Theorem 7.1 of Shapiro (2016)).

Let $y^{m}=\gamma^{m}\left(x^{m}\right)$. Since $X \times Y$ is compact, the sequence $\left(x^{m}, y^{m}\right)$ converges to some $(x, y) \in X \times Y$, perhaps after taking a subsequence. By standard arguments,

$$
(x, y) \in \operatorname{graph}(\tilde{K})
$$

for some $y \in Y$. And so

$$
\left(x^{m}, x^{m}\right)=\left(x^{m}, H\left(x^{m}, y^{m}\right)\right) \rightarrow(x, H(x, y)),
$$

which implies $x \in K(x)$, completing the proof.

To show existence of an equilibrium, it only remains to show that $G$ is a convex, compact, closed correspondence, which follows immediately from the following.

Lemma 7. The correspondence $\mathcal{C}(v)$ has a closed graph. 
Proof. Consider a sequence $\left(v^{m}, \Phi^{m}\right)$ for which $\Phi^{m} \in \mathcal{C}\left(v^{m}\right)$ and $\left(v^{m}, \Phi^{m}\right) \rightarrow(v, \Phi)$. We claim that $\int_{M_{i}(v)^{c}} d \phi_{i}=0$. For any positive integer $k$, set

$$
O_{k}=\bigcup_{j \neq i}\left\{x:\left(v^{j}\right)^{-1}\left[1-F^{j}(x)\right]-\left(v^{i}\right)^{-1}\left[1-F^{i}(x)\right]>1 / k\right\} .
$$

When $m$ is sufficiently large, $O_{k} \subseteq M_{i}\left(v^{m}\right)^{c}$ as, otherwise, the closure of $O_{k}$ and $M_{i}(v)$ has a nonempty intersection. Hence, $\int_{O_{k}} 1 d \phi_{i}^{m}=0$ for $m$ sufficiently large, and thus, $\int_{O_{k}} 1 d \phi_{i}=0 .^{21}$ Since $O_{k} \uparrow M_{i}(v)^{c}$, the monotone convergence theorem implies $\int_{M_{i}(v)^{c}} d \phi_{i}=0$.

Thus, an equilibrium exists.

Lемма 8. With risk neutrality, the equilibrium asset prices and utility of each agent are unique.

Proof. Consider equilibrium allocations $\left(\hat{a}^{j, 0}, \hat{\mu}^{j, 0} ;\left(\hat{a}^{j, i}, \hat{c}^{j, i}, \hat{\mu}^{j, i}\right)_{i=1}^{N}\right)$ at prices $\left(p^{j}, q^{j}\right)$ for $j=1,2$ with return vectors $v^{1}=\left(v^{1,1}, \ldots, v^{1, N}\right)$ and $v^{2}=\left(v^{2,1}, \ldots, v^{2, N}\right)$, respectively. $^{22}$ We can assume without loss that $\hat{a}^{j, i}=0$ for $i=1, \ldots, N$, that $\hat{a}^{j, 0}=e^{a}$ for $j=1,2$, and that each security purchased is right continuous. If the result is false, then $v^{1} \neq v^{2}$; otherwise, the price and utility are equal by Lemmas 1 and 2. Let $\eta^{i}$ and $\zeta^{i}$ be the Lesbesgue-Stieltjes measures of $\int_{\Sigma} \phi d \hat{\mu}^{1, i}$ and $\int_{\Sigma} \phi d \hat{\mu}^{2, i}$ for each $i=1, \ldots, N$.

Let $c=\max _{i}\left\{v^{1, i} / v^{2, i}\right\}>1$. Note that $c v^{2, i} \geq v^{1, i}$ for each $i$ and that $r(\cdot)$ is homogeneous of degree -1 . For each $i$ and all vectors of $N$ increasing, right-continuous functions $\gamma$ and $z \in[1, \infty)^{N}$, define

$$
g_{i}(z, \gamma)=\frac{\int_{S} \widetilde{F}^{i}(x) d \gamma^{i}}{e_{c}^{i}+r(z) e_{a}^{i}}
$$

and let $g_{i}^{*}(z, \gamma)=\max \left\{g_{i}(z, \gamma), 1\right\}$. In equilibrium, we must have $v^{1, i}=g_{i}^{*}\left(v^{1}, \eta\right)$ and $v^{2, i}=g_{i}^{*}\left(v^{2}, \zeta\right)$ for all $i$ as above.

For any $d>1$, any $z \in[1, \infty)^{N}$, and any Trader $i$, we have

$$
\frac{1}{d} g_{i}(d z, \eta)=\frac{\int_{S} \widetilde{F}^{i}(x) d \eta^{i}}{d e_{c}^{i}+d r(d z) e_{a}^{i}}=\frac{\int_{S} \widetilde{F}^{i}(x) d \eta^{i}}{d e_{c}^{i}+r(z) e_{a}^{i}}<g_{i}(z, \eta)
$$

and so $\frac{1}{d} g_{i}^{*}(d z, \eta)=\max \left\{\frac{1}{d}, \frac{1}{d} g_{i}(d z, \eta)\right\}<\max \left\{1, g_{i}(z, \eta)\right\}=g_{i}^{*}(z, \eta)$ for all $i$ and all $d>$ 1. Thus, for each Trader $i$,

$$
v^{2, i}=g_{i}^{*}\left(v^{2}, \zeta\right)>c^{-1} g_{i}^{*}\left(c v^{2}, \zeta\right)
$$

${ }^{21}$ Since $\psi_{i}^{m} \rightarrow \psi$ pointwise everywhere, it follows that $\psi_{i}^{m}$ converges weakly to $\psi$ as a distribution by Ash and Doleans, Theorem 2.8.4(b). Since $O_{k}$ is open, the claim follows by Ash and Doleans, Theorem 2.8.1(d).

${ }^{22}$ Note the first superscript denotes which equilibrium and the second the identity of the trader. 
Define $M_{i}(\cdot)$ as in equation (15). Since $M_{i}\left(c v^{2}\right)=M_{i}\left(v^{2}\right)$ for every $i \in I, \int_{M_{i}\left(c v^{2}\right)^{c}} d \eta^{i}=0$. Let $I^{\prime}$ be the set $\left\{i: v^{1, i}=c v^{2, i}\right\}$ and note that $I^{\prime} \neq \emptyset$ by construction and $I^{\prime} \neq\{1, \ldots, N\}$ since $v^{1, j}=1$ for some Trader $j$. Consider $x \in M_{i}\left(v^{1}\right) \cap[0, s), i \in I^{\prime}$, and $k \notin I^{\prime}$. Since $x \in M_{i}\left(v^{1}\right) \cap[0, s), \widetilde{F}^{i}(x)>0$ and when $\widetilde{F}^{k}(x)>0$,

$$
\left(c v^{2, k}\right)^{-1} \widetilde{F}^{k}(x)<\left(v^{1, k}\right)^{-1} \widetilde{F}^{k}(x) \leq\left(v^{1, i}\right)^{-1} \widetilde{F}^{i}(x)=\left(c v^{2, i}\right)^{-1} \widetilde{F}^{i}(x) .
$$

When $\widetilde{F}^{k}(x)=0$, obviously $\left(c v^{2, k}\right)^{-1} \widetilde{F}^{k}(x)<\left(c v^{2, i}\right)^{-1} \widetilde{F}^{i}(x)$ and so $x \notin M_{k}\left(c v^{2}\right)$ and $\int_{M_{i}\left(v^{1}\right)} d \zeta^{k}=0$. Moreover, if $x \in M_{i}\left(v^{1}\right)$, then $x \in M_{i}\left(c v^{2}\right)$. Since $\sum_{i=1}^{N} \int_{E} d \eta^{i}=$ $\sum_{i=1}^{N} \int_{E} d \zeta^{i}=\lambda(E)$ for all Borel $E \subset S$, when $E=\bigcup_{i \in I^{\prime}} M_{i}\left(v^{1}\right)$ we have

$$
\begin{gathered}
\sum_{i^{\prime} \in I^{\prime}} \int_{E}\left(v^{1, i^{\prime}}\right)^{-1} \widetilde{F}^{i^{\prime}} d \eta^{i^{\prime}} \leq \sum_{i^{\prime} \in I^{\prime}} \int_{E}\left(c v^{2, i^{\prime}}\right)^{-1} \widetilde{F}^{i^{\prime}}(x) d \zeta^{i^{\prime}} \\
\sum_{i^{\prime} \in I^{\prime}} \int_{E^{c}}\left(v^{1, i^{\prime}}\right)^{-1} \widetilde{F}^{i^{\prime}} d \eta^{i^{\prime}}=0 \leq \sum_{i^{\prime} \in I^{\prime}} \int_{E^{c}}\left(c v^{2, i^{\prime}}\right)^{-1} \widetilde{F}^{i^{\prime}}(x) d \zeta_{i^{\prime}} .
\end{gathered}
$$

Conclude

$$
\sum_{i \in I^{\prime}} \int_{S} \widetilde{F}^{i}(x) d \zeta^{i} \geq \sum_{i \in I^{\prime}} \int_{S} \widetilde{F}^{i}(x) d \eta^{i}
$$

and so for some $i^{*} \in I^{\prime}$, we must have

$$
\int_{S} \widetilde{F}^{i^{*}}(x) d \zeta^{i^{*}} \geq \int_{S} \widetilde{F}^{i^{*}}(x) d \eta^{i^{*}}
$$

Since $c v^{2} \geq v^{1}$ implies $r\left(c v^{2}\right) \leq r\left(v^{1}\right)$, we have $g_{i^{*}}^{*}\left(c v^{2}, \zeta\right) \geq g_{i^{*}}^{*}\left(v^{1}, \eta\right)$, and thus

$$
v^{2, i^{*}}>c^{-1} g_{i^{*}}^{*}\left(c v^{2} ; \zeta\right) \geq c^{-1} g_{i^{*}}^{*}\left(v^{1}, \eta\right)=c^{-1} v^{1, i^{*}}=v^{2, i^{*}},
$$

a contradiction.

\section{A.4 Other proofs}

Proof of Corollary 1. Consider an equilibrium allocation $\left(\hat{a}^{0}, \hat{\mu}^{0} ;\left(\hat{a}^{i}, \hat{c}^{i}, \hat{\mu}^{i}\right)_{i=1}^{N}\right)$ with return vector $\hat{v}$ and $\hat{w}=s \mapsto\left(\hat{a}^{i} s+\hat{c}^{i}+\int \phi d \hat{\mu}^{i}\right)_{i=1}^{N}$. Normalize $e^{a}=1$ for simplicity. As above, we can assume without loss that $\hat{a}^{i}=0$ for $i=1, \ldots, N$ and $\hat{a}^{0}=e^{a}$. By Theorem 2 , utility is unique so the return vector is the same in any equilibrium. So too are the sets

$$
M_{i}^{\circ}=\left\{x \in S:\left(\hat{v}^{i}\right)^{-1} \widetilde{F}^{i}(x)>\left(\hat{v}^{k}\right)^{-1} \widetilde{F}^{k}(x) \forall k \neq i\right\}
$$

for $i=1, \ldots, N$. Observe that for any $(a, b) \subseteq M_{i}^{\circ}, \hat{w}_{i}(b)-\hat{w}_{i}(a)=b-a$. Under Assumption 1, $\bigcup_{i=1}^{N} M_{i}^{\circ}$ contains all but finitely many points in $S$, and each $M_{i}^{\circ}$ is a finite collection of intervals. Feasibility of securities then implies that $\hat{w}_{i}$ must also be unique, since a jump at any of the points not in one of the $M_{i}^{\circ}$ 's necessarily decreases revenue.

Define $\hat{\Phi}=\left(\hat{\phi}_{1}, \ldots, \hat{\phi}_{N}\right)$ so that $\hat{\phi}_{i}=\int_{\Sigma} \phi d \hat{\mu}^{i}$. Now, there exist $K$ intervals $\left\{\left(b_{j}\right.\right.$, $\left.\left.b_{j+1}\right)\right\}_{j=1}^{K}$ with $b_{1}=0, b_{K}=\bar{s}$ and $b_{j+1}>b_{j}$ such that for every $j=1, \ldots, K$ there exist 
$i \in\{1, \ldots, N\}$ so that $\left(b_{j}, b_{j+1}\right) \subset M_{i}^{\circ}$ and $M_{i}^{\circ} \bigcap\left[\left(b_{j-1}, b_{j}\right) \bigcup\left(b_{j+1}, b_{j+2}\right)\right]=\emptyset$. Since $\hat{\phi}_{i}$ is continuous, $\hat{\phi}_{i}(0)=0$ for every $i=1, \ldots, N$, and $\hat{\phi}_{I}$ increases only on $M_{i}^{\circ}$, we can take $\mu^{0}=a^{0} \sum_{j=1}^{K} \delta_{\left\{\phi_{\left[b_{j}, b_{j+1}\right]}\right\}}$ and $\mu^{i}=a^{0} \sum_{\left(b_{j}, b_{j+1}\right) \subset M_{i}^{\circ}} \delta_{\left\{\phi_{\left[b_{j}, b_{j+1}\right]}\right\}}$ to obtain an equivalent equilibrium that is tranching and sorting.

Proof of Corollary 2. Consider an equilibrium return vector $\hat{v}$ and an equilibrium allocation $\left(\hat{a}^{0}, \hat{\mu}^{0} ;\left(\hat{a}^{i}, \hat{\mu}^{i}, \hat{c}^{i}\right)\right)$. First, we claim that $\hat{v}^{i}=1$ for at least one $i$. If $\hat{v}^{i}>1$ for all $i$, then $\left(\hat{v}^{i}\right)^{-1} \mathbb{E}^{i}\left[\int \phi d \hat{\mu}^{i}\right] e^{a}=e_{c}^{i}+\hat{p} e_{a}^{i}$ for all $i$. Recall $\hat{p}=\sum_{i=1}^{N}\left(\hat{v}^{i}\right)^{-1} \mathbb{E}^{i}\left[\int \phi d \hat{\mu}^{i}\right]$. Summing across $i$, we have $\hat{p} e^{a}=\sum_{i} e_{c}^{i}+\hat{p} e^{a}$, a contradiction. Thus, there exists $i^{*}$ with $\hat{v}^{i^{*}}=1$. Then $\hat{p}=\int \max _{k}\left(v^{k}\right)^{-1} \widetilde{F}^{k}(x) d x \geq \int\left(\hat{v}^{i^{*}}\right)^{-1} \widetilde{F}^{i^{*}}(x) d x=m$.

Now, suppose Assumption 1 holds. If $\int q d \mu_{j}=0$ for all $j \neq i^{*}$, then $\left(v^{j}\right)^{-1}=1$ for all $j$; all agents have positive wealth and so must be purchasing the safe asset. Then $\lambda\left(\left\{x: \widetilde{F}^{j}(x)>\widetilde{F}^{i}(x)\right\}\right)>0$ since otherwise $\int \widetilde{F}^{j}(x) d x=\int \widetilde{F}^{i}(x) d x$ implies $\widetilde{F}^{j}(x)=\widetilde{F}^{i}(x)$ for almost all $x$, contradicting Assumption 1. Hence, $\int q d \mu_{i^{*}}, \int q d \mu_{j}>0$ for some distinct $j, i^{*} \in\{1, \ldots, N\}$. Then the set $\left\{x:\left(v^{j}\right)^{-1} \widetilde{F}^{j}(x) \geq \widetilde{F}^{i^{*}}(x)\right\}$ has positive measure, and by Assumption 1, so does $B=\left\{x:\left(v^{j}\right)^{-1} \widetilde{F}^{j}(x)>\widetilde{F}^{i^{*}}(x)\right\}$. Conclude $\hat{p} \geq$ $\int_{B}\left(v^{j}\right)^{-1} \widetilde{F}^{j}(x) d x+\int_{B^{c}} \widetilde{F}^{i}(x) d x>m$.

\section{Appendix B: Details and Proofs from Section 5}

\section{B.1 Definition of pooling equilibrium}

Trader $i$ 's purchases of securities backed by asset $j$ is described by $\mu_{j}^{i} \in \mathcal{M}(\Sigma)$, of securities backed by pool $\pi^{k}$ by $\mu_{k}^{i} \in \mathcal{M}(\Sigma)$, and of asset $j$ by $a_{j}^{i}$. Then trader $i$ 's expected utility $V^{i}\left(c^{i}, a^{i}, \mu^{i}\right)$ is

$$
c^{i}+\mathbb{E}^{i}\left[a^{i} \cdot s\right]+\sum_{j=1}^{J} \int_{\Sigma} \int_{S} \phi\left(s_{j}\right) d F_{j}^{i}\left(s_{j}\right) d \mu_{j}^{i}(\phi)+\sum_{k>J} \int_{\Sigma} \int_{S} \phi(x) d F^{i}\left(x ; \pi^{k}\right) d \mu_{k}^{i}(\phi) .
$$

The purchases $\left(c^{i}, a^{i}, \mu^{i}\right)$ maximize utility for prices $(p, q)$ and wealth $w(p, q)=p \cdot e^{i}+$ $e_{c}^{i}$ if

$$
V^{i}\left(c^{i}, a^{i}, \mu^{i}\right)=\max _{j, \phi, \pi}\left\{w(p, q), \frac{w(p, q)}{q(\phi,\{j\})} \int_{S} \phi\left(s_{j}\right) d F_{j}^{i}\left(s_{j}\right), \frac{w(p, q)}{q(\phi, \pi)} \int_{S} \phi(x) d F^{i}(x ; \pi)\right\} .
$$

An allocation $\left(\left(\hat{a}^{0}, \hat{\mu}^{0}\right) ;\left(\hat{c}^{i}, \hat{a}^{i}, \hat{\mu}^{i}\right)_{i=1}^{N}\right)$ is an equilibrium for $(\hat{p}, \hat{q})$, if $\left(\hat{a}^{0}, \hat{\mu}^{0}\right)$ maximizes profit for $(\hat{p}, \hat{q}),\left(\hat{a}^{i}, \hat{\mu}^{i}\right)$ maximizes utility for $(\hat{p}, \hat{q})$ for each $i$, and is attainable:

$$
\begin{aligned}
\sum_{i=0}^{N} \hat{a}_{j}^{i} & =\sum_{i=1}^{N} e_{j}^{i} \quad \forall j=1, \ldots, J \\
\hat{\mu}_{j}^{0} & =\sum_{i} \hat{\mu}_{j}^{i} \quad \forall j=1, \ldots, 2 J \\
\sum_{i=1}^{N} \hat{c}^{i} & =\sum_{i=1}^{N} e_{c}^{i} .
\end{aligned}
$$


REMARK 4. We note that the distinction between securities based on individual assets and those based on pools is redundant. We can replicate any single asset securities by those based on a pool containing only that asset. The distinction is maintained for expositional clarity of the scope of options available to the issuer.

\section{B.2 Proofs}

B.2.1 Proof of Theorem 3 Let $\Delta(\Pi)$ be the set of Borel probability measures on $\Pi$, with the weak*-topology. Define

$$
\begin{aligned}
R(\pi) & =\int_{0}^{\bar{s}} \max _{i} \widetilde{F}^{i}(x ; \pi) d x, \\
\Gamma(p) & =\arg \max _{z \in \Delta(\Pi)} \int[R(\pi)-\pi \cdot p] d z(\pi) \\
\bar{p}_{j} & =\int_{S} \max _{i} \widetilde{F}_{j}^{i}(x) d x
\end{aligned}
$$

where $\bar{p}=\left(\bar{p}_{1}, \ldots, \bar{p}_{J}\right)$ is the revenue from optimally securitizing each asset individually. The set $\Gamma(p)$ is the set of distributions over the normalized pools that achieve maximum profits. The correspondence $\Gamma(p)$ is nonempty and convex valued and has a closed graph since $\Delta \Pi$ is weak*-compact.

Given $z \in \Delta(\Pi)$, define

$$
\begin{aligned}
\beta(z) & =\min _{j} \frac{e_{j}}{\int \pi_{j} d z(\pi)}, \\
P & =\left\{p \in \mathbb{R}_{+}^{J}: \bar{p} \leq p \leq\left(\max _{\pi} R(\pi) \sum_{j=1}^{J} e_{j}\right)\left(e_{1}^{-1}, e_{2}^{-1}, \ldots, e_{J}^{-1}\right)\right\}
\end{aligned}
$$

and

$\Phi(z)=\left\{\begin{array}{l}\{\bar{p}\} \quad \text { if } \int(R(\pi)-\bar{p} \cdot \pi) d z(\pi) \leq 0 \\ \left\{q \geq \bar{p}: 0=\int(R(\pi)-q \cdot \pi) d z(\pi) \& q_{j}=\bar{p}_{j} \text { if } e_{j}>\beta(z) \int \pi_{j} d z\right\} \text { otherwise } .\end{array}\right.$

Note that $\Phi(z)$ is a nonempty, convex subset of $P$, and that $\Phi$ has a closed graph. The convexity of $\Phi(z)$ for any $z \in \Delta(\Pi)$ is obvious. To see that $\Phi(z)$ is nonempty, suppose that $\int(R(\pi)-\bar{p} \cdot \pi) d z(\pi)>0$. Since $e_{j}=\beta(z) \int \pi_{j} d z$ for some $j$ by construction, $q \in \mathbb{R}_{+}^{J}$ such that $q_{k}=\bar{p}_{k}$ for $j \neq k$ and

$$
q_{j}=\left(\int\left(R(\pi)-\sum_{k \neq j} \bar{p}_{k} \pi_{k}\right) d z(\pi)\right)\left(\int \pi_{j} d z(\pi)\right)^{-1}
$$

is such that $q_{j} \geq \bar{p}_{j}$. Hence, $q \in \phi(z)$. 
To see that $\Phi(z) \subset P$, if $q \in \Phi(z)$ and $q_{j}>\bar{p}_{j}$, then $e_{j}=\beta(z) \alpha_{j}$ where $\alpha=\int \pi d z(\pi)$. Since for all $k \in J, e_{k} \geq \beta(z) \alpha_{k}$,

$$
\alpha_{j}=\frac{\beta(z) \alpha_{j}}{\sum_{k=1}^{J} \beta(z) \alpha_{k}} \geq \frac{e_{j}}{\sum_{k=1}^{J} e_{k}}
$$

and thus,

$$
q_{j} \leq \frac{\int R(\pi) d z(\pi)}{\alpha_{j}} \leq \int R(\pi) d z(\pi)\left(\sum_{k=1}^{J} e_{k}\right) e_{j}^{-1} .
$$

It is straightforward to show that $\Phi$ has a closed graph.

By the Kakutani-Fan-Glicksburg theorem, there exists $(p, z)$ such that $(p, z) \in$ $\Phi(z) \times \Gamma(p)$. At this fixed point, $R(\pi)=\pi \cdot p$ for $z$-a.e. $\pi$ since, letting $R_{j}$ denote $R(\pi)$ when $\pi_{j}=1$ and $\pi_{k}=0$ for all other $k$ we have $R_{j}-\bar{p}_{j}=0$. Moreover, selling $\beta(z) z(\pi)$ units of each $\pi$ achieves this profit, and does not exceed the total endowment. Whenever $\int \beta(z) \pi_{j} z(\pi)<e_{j}$, the firm does not use all of asset $j$ in pooling, and the traders hold securities based on the remaining units. This also achieves zero profit since $\bar{p}_{j}=p_{j}$ is the revenue from optimally securitizing one unit of asset $j$.

Finally, note that $\int \pi d z \in c o\left(\arg \max _{\pi} R(\pi)-\pi \cdot p\right)$. Since $\Pi$ has dimension $J-1$, there are $\pi^{J+1}, \ldots, \pi^{2 J} \in \arg \max _{\pi} R(\pi)-\pi \cdot p$ and $\gamma^{J+1}, \ldots, \gamma^{2 J} \geq 0$ so that $\sum_{k=J+1}^{2 J} \gamma^{k} \pi^{k}=\int \pi d z$ and $\sum_{k=J+1}^{2 J} \gamma^{k}=1$ by Caratheodory's theorem. Since $(z, p) \in$ $\Phi(z) \times \Gamma(p)$, each $\pi^{k}$ obtains zero profit and no other pool obtains positive profit. The issuer securitizes $a_{k}^{0}=\gamma^{k} \beta(z)$ for $k>J$ units of pool $k$, and the residual asset of $j$ is securitized on its own: $a_{j}^{0}=e_{j}-\beta(z) \int \pi_{j} d z$. This maximizes profit subject to the constraints.

B.2.2 Proof of Theorem 4 For $s \in \mathbb{R}^{J}$, we follow standard notational conventions by denoting $s_{-i}$ for the element of $\mathbb{R}^{J-1}$ that drops the $i$ th coordinate. We can write the $\operatorname{CDF} F^{i}(\cdot ; \pi)$ as

$$
F^{i}(z ; \pi)=\int_{\mathbb{R}^{J-1}} C_{-k}\left(F_{k}^{i}\left(\pi_{k}^{-1}\left(z-\sum_{j \neq k} \pi_{j} s_{j}\right)\right) ;\left(F_{j}^{i}\left(s_{j}\right)\right)_{j \neq k}\right) \prod_{j \neq k} f_{j}^{i}\left(s_{j}\right) d s^{-k}
$$

where $C_{-k}\left(x_{k} ; x_{-k}\right)=\frac{\partial^{J-1} C\left(x_{1}, \ldots, x_{J}\right)}{\prod_{j \neq k} \partial x_{j}}$ for any $k=1, \ldots, J$. Obviously, $F_{j}^{m}(x)=\min _{i} F_{j}^{i}(x)$ is a well defined CDF and has a density $f_{j}^{m}$ almost everywhere for each $j$. Since $\frac{\partial^{J} C\left(x_{1}, \ldots, x_{J}\right)}{\prod_{j=1}^{J} \partial x_{j}} C(x) \geq 0$,

$$
\begin{aligned}
F^{i}(z ; \pi) & \geq \int_{\mathbb{R}^{J-1}} C_{-1}\left(F_{1}^{m}\left(\pi_{1}^{-1}\left(z-\sum_{j \neq 1} \pi_{j} s_{j}\right)\right) ;\left(F_{j}^{i}\left(s_{j}\right)\right)\right) \prod_{j>1} f_{j}^{i}\left(s_{j}\right) d s_{-1} \\
& =\int_{\mathbb{R}^{J-1}} C_{-2}\left(F_{2}^{i}\left(\pi_{2}^{-1}\left(z-\sum_{j \neq 2} \pi_{j} s_{j}\right)\right) ; F_{1}^{m}\left(s^{1}\right),\left(F_{j}^{i}\left(s_{j}\right)\right)_{j>2}\right) f_{1}^{m}\left(s_{1}\right) \prod_{j>2} f_{j}^{i}\left(s_{j}\right) d s_{-2}
\end{aligned}
$$




$$
\begin{aligned}
& \geq \int_{\mathbb{R}^{J-1}} C_{-2}\left(F_{2}^{m}\left(\pi_{2}^{-1}\left(z-\sum_{j \neq 2} \pi_{j} s_{j}\right)\right) ; F_{1}^{m}\left(s^{1}\right),\left(F_{j}^{i}\left(s_{j}\right)\right)_{j>2}\right) f_{1}^{m}\left(s_{1}\right) \prod_{j>2} f_{j}^{i}\left(s_{j}\right) d s_{-2} \\
& \geq \cdots \geq \int_{\mathbb{R}^{J-1}} C_{-J}\left(F_{J}^{m}\left(\pi_{J}^{-1}\left(z-\sum_{j<J} \pi_{j} s_{j}\right)\right) ;\left(F_{j}^{m}\left(s_{j}\right)\right)_{j<J}\right) \prod_{j<J} f_{j}^{m}\left(s_{j}\right) d s_{-J}
\end{aligned}
$$

where the subsequent inequalities are obtained repeating the steps above for assets $3, \ldots, J$. It follows that the $\operatorname{CDF} F^{m}(\cdot ; \pi)$ first-order stochastically dominates each $F^{i}(\cdot ; \pi)$ for $i=1, \ldots, N$. Since the expected value of the sum of random variables is the sum of their expected values,

$$
\int_{0}^{\infty} \widetilde{F}^{m}(z ; \pi) d z=\sum_{j=1}^{J} \pi_{j} \int_{0}^{\infty} \widetilde{F}_{j}^{m}(z) d z=\sum_{j=1}^{J} \pi^{j} \int_{0}^{\infty} \max _{i} \widetilde{F}_{j}^{i}(z) d z .
$$

Thus, by the definition of $q(\phi, \pi)$ the revenue for any securitization of the pool $\pi$ is bounded by the revenue from the optimal securitization of individual assets.

Now, assume that traders' beliefs about $s_{j}$ satisfy the finite crossing condition and $F_{j}^{i} \neq F_{j}^{m}$ for every $i$. We show that there is no equilibrium where $\pi_{j}^{k} \in(0,1)$ and $a_{k}^{0}>0$. Relabel so that $j=1$.

Consider traders $i \neq i^{\prime}, x \in(0, \bar{s})$, and $\epsilon>0$ so that $F_{1}^{i}(y)=F_{1}^{m}(y)$ for $y \in(x-\epsilon, x)$ and $F_{i^{\prime}}^{1}(y)=F_{1}^{m}(y)$ for $y \in(x, x+\epsilon)$. Such an $x$ exists because $F_{1}^{i} \neq F_{1}^{m}$ for each $i$. By finite crossing, $F_{i^{\prime}}^{1}(y)=F_{1}^{m}(y) \leq F_{k}^{1}(y)$ for $y \in(x, x+\epsilon)$ when $k \neq i^{\prime}$ and $F_{k}^{1}(y) \geq F_{1}^{i}(y)=F_{1}^{m}(y)$ for $y \in(x-\epsilon, x)$ when $k \neq i$. For any $\pi$ with $\pi_{1} \in(0,1)$,

$$
\begin{aligned}
& F^{i^{\prime \prime}(x ; \pi)} \\
& =\int_{\left\{s^{-1}: \sum_{j \neq 1} \pi_{j} s_{j} \in(x, x+\epsilon)\right\}} C_{-1}\left(F_{1}^{i^{\prime \prime}}\left(\pi_{1}^{-1}\left(x-\sum_{j \neq 1} \pi_{j} s_{j}\right)\right) ;\left(F_{j}^{i^{\prime \prime}}\left(s_{j}\right)\right)_{j>1}\right) \prod_{j>1} f_{j}^{i^{\prime \prime}}\left(s_{j}\right) d s_{-1} \\
& \quad+\int_{\left\{s^{-1}: \sum_{j \neq 1} \pi_{j} s_{j} \in(x-\epsilon, x)\right\}} C_{-1}\left(F_{1}^{i^{\prime \prime}}\left(\pi_{1}^{-1}\left(x-\sum_{j \neq 1} \pi_{j} s_{j}\right)\right) ;\left(F_{j}^{i^{\prime \prime}}\left(s_{j}\right)\right)_{j>1}\right) \prod_{j>1} f_{j}^{i^{\prime \prime}}\left(s_{j}\right) d s_{-1} \\
& \quad+\int_{\left\{s^{-1}: \sum_{j \neq 1} \pi_{j} s_{j} \notin(x-\epsilon, x+\epsilon)\right\}} C_{-1}\left(F_{1}^{i^{\prime \prime}}\left(\pi_{1}^{-1}\left(x-\sum_{j \neq 1} \pi_{j} s_{j}\right)\right) ;\left(F_{j}^{i^{\prime \prime}}\left(s_{j}\right)\right)_{j>1}\right) \\
& \quad \times \prod_{j>1} f_{j}^{i^{\prime \prime}}\left(s_{j}\right) d s_{-1}
\end{aligned}
$$

and as above

$$
\begin{aligned}
& \int_{E} C_{-1}\left(F_{1}^{i^{\prime \prime}}\left(\pi_{1}^{-1}\left(x-\sum_{j \neq 1} \pi_{j} s_{j}\right)\right) ;\left(F_{j}^{i^{\prime \prime}}\left(s_{j}\right)\right)_{j>1}\right) \prod_{j>1} f_{j}^{i^{\prime \prime}}\left(s_{j}\right) d s_{-1} \\
& \geq \int_{E} C_{-1}\left(F_{1}^{m}\left(\pi_{1}^{-1}\left(z-\sum_{j \neq 1} \pi_{j} s_{j}\right)\right) ;\left(F_{j}^{i^{\prime \prime}}\left(s_{j}\right)\right)_{j>1}\right) \prod_{j>1} f_{j}^{i^{\prime \prime}}\left(s_{j}\right) d s_{-1}
\end{aligned}
$$


for any measurable $E \subset S^{J-1}$. Moreover, If $i^{\prime \prime} \neq i$, then

$$
\begin{gathered}
\int_{\left\{s^{-1}: \sum_{j \neq 1} \pi_{j} s_{j} \in(x-\epsilon, x)\right\}} C_{-1}\left(F_{1}^{i^{\prime \prime}}\left(\pi_{1}^{-1}\left(x-\sum_{j \neq 1} \pi_{j} s_{j}\right)\right) ;\left(F_{j}^{i^{\prime \prime}}\left(s_{j}\right)\right)_{j>1}\right) \prod_{j>1} f_{j}^{i^{\prime \prime}}\left(s_{j}\right) d s_{-1} \\
>\int_{\left\{s^{-1}: \sum_{j \neq 1} \pi_{j} s_{j} \in(x-\epsilon, x)\right\}} C_{-1}\left(F_{1}^{m}\left(\pi_{1}^{-1}\left(z-\sum_{j \neq 1} \pi_{j} s_{j}\right)\right) ;\left(F_{j}^{i^{\prime \prime}}\left(s_{j}\right)\right)_{j>1}\right) \prod_{j>1} f_{j}^{i^{\prime \prime}}\left(s_{j}\right) d s_{-1} .
\end{gathered}
$$

since $\frac{\partial}{\partial x_{1}} C_{-1}(x)>0$ and $F_{1}^{m}(y)<F_{1}^{i^{\prime \prime}}(y)$ for $y \in(x-\epsilon, x)$. Similarly, if $i^{\prime \prime} \neq i^{\prime}$, then

$$
\begin{aligned}
& \int_{\left\{s^{-1}: \sum_{j \neq 1} \pi_{j} s_{j} \in(x, x+\epsilon)\right\}} C_{-1}\left(F_{1}^{i^{\prime \prime}}\left(\pi_{1}^{-1}\left(x-\sum_{j \neq 1} \pi_{j} s_{j}\right)\right) ;\left(F_{j}^{i^{\prime \prime}}\left(s_{j}\right)\right)_{j>1}\right) \prod_{j>1} f_{j}^{i^{\prime \prime}}\left(s_{j}\right) d s_{-1} \\
& >\int_{\left\{s^{-1}: \sum_{j \neq 1} \pi_{j} s_{j} \in(x-\epsilon, x)\right\}} C_{-1}\left(F_{1}^{m}\left(\pi_{1}^{-1}\left(z-\sum_{j \neq 1} \pi_{j} s_{j}\right)\right) ;\left(F_{j}^{i^{\prime \prime}}\left(s_{j}\right)\right)_{j>1}\right) \prod_{j>1} f_{j}^{i^{\prime \prime}}\left(s_{j}\right) d s_{-1} .
\end{aligned}
$$

Since $i^{\prime} \neq i$, we have strict inequality in the first step of the above sequence of inequalities. Conclude that the $\operatorname{CDF} F^{m}(\cdot ; \pi)$ strictly first-order stochastically dominates each $F^{i}(\cdot ; \pi)$ for $i=1, \ldots, N$. As above, integrating the former obtains the revenue from individually securitizing the assets in $\pi$ individually.

B.2.3 Proof of Theorem 5 Relabel so that $j=1$ and $j^{\prime}=2$. Consider the pool $\pi^{k}=$ $(\pi,(1-\pi), 0, \ldots)$. Then, as $\pi \rightarrow 0$, we have

$$
\begin{aligned}
\frac{d}{d \pi} & \widetilde{F}^{i}\left(x ; \pi^{k}\right) \\
& =-\int_{0}^{\bar{s}} \frac{d}{d \pi} \frac{\partial}{\partial x_{1}} C^{i}\left(F_{1}(y), F_{2}\left(\frac{x-\pi y}{1-\pi}\right), 1, \ldots\right) f_{1}(y) d y \\
& =-\int_{0}^{\bar{s}} \frac{\partial^{2}}{\partial x_{1} \partial x_{2}} C^{i}\left(F_{1}(y), F_{2}\left(\frac{x-\pi y}{1-\pi}\right), 1, \ldots\right) f_{1}(y) f_{2}\left(\frac{x-\pi y}{1-\pi}\right) \frac{x-y}{(1-\pi)^{2}} d y \\
& \rightarrow-\int_{0}^{\bar{s}} \frac{\partial^{2}}{\partial x_{1} \partial x_{2}} C^{i}\left(F_{1}(y), F_{2}(x), 1, \ldots\right) f_{2}(x) f_{1}(y)(x-y) d y \\
& =-f_{2}(x) \int_{0}^{\bar{s}} \frac{\partial^{2}}{\partial x_{1} \partial x_{2}} C^{i}\left(F_{1}(y), F_{2}(x), 1, \ldots\right) f_{1}(y)(x-y) d y \\
& =-f_{2}(x)\left[x-\mathbb{E}^{i}\left[s_{1} \mid s_{2}=x\right]\right]
\end{aligned}
$$

since for a.e. $x \in S$,

$$
\mathbb{E}^{i}\left[s_{1} \mid s_{2}=x\right]=\int_{0}^{\bar{s}} y \frac{\partial^{2}}{\partial x_{1} \partial x_{2}} C^{i}\left(F_{1}(y), F_{2}(x), 1, \ldots\right) f_{1}(y) d y
$$

is Trader $i$ 's conditional expectation of $s_{1}$ given $s_{2}=x$. If $\mathbb{E}^{i^{\prime}}\left[s_{1} \mid s_{2}=x\right]>\mathbb{E}^{i}\left[s_{1} \mid s_{2}=x\right]$ for some Traders $i, i^{\prime}$ and $x \in(0, \bar{s})$, then there is a neighborhood $O \ni x$ so that $\mathbb{E}^{i^{\prime}}\left[s_{1} \mid s_{2}=\right.$ $x]>\mathbb{E}^{i}\left[s_{1} \mid s_{2}=x\right]$ for all $x^{\prime} \in O$. For $\pi$ sufficiently close to $0, \widetilde{F}^{i^{\prime}}\left(x ; \pi_{k}\right)-\widetilde{F}^{i}\left(x ; \pi_{k}\right)>0$ for 
all $x \in O$, and hence

$$
\begin{aligned}
\int_{0}^{\bar{s}} \max _{i^{\prime \prime}} \widetilde{F}^{i^{\prime \prime}}\left(x ; \pi_{k}\right) d x & =\int_{0}^{\bar{s}} \widetilde{F}^{i}\left(x ; \pi_{k}\right) d x+\int_{0}^{\bar{s}} \max \left\{\widetilde{F}_{i^{\prime \prime}}^{i^{\prime \prime}}\left(x ; \pi_{k}\right)-\widetilde{F}^{i}\left(x ; \pi_{k}\right)\right\} d x \\
& \geq \int_{0}^{\bar{s}} \widetilde{F}^{i}\left(x ; \pi_{k}\right) d x+\int_{0}^{\bar{s}} \max \left\{\widetilde{F}^{i^{\prime}}\left(x ; \pi_{k}\right)-\widetilde{F}^{i}\left(x ; \pi_{k}\right), 0\right\} d x \\
& \geq \int_{0}^{\bar{s}} \widetilde{F}^{i}\left(x ; \pi_{k}\right) d x+\int_{O}\left[\widetilde{F}^{i^{\prime}}\left(x ; \pi_{k}\right)-\widetilde{F}^{i}\left(x ; \pi_{k}\right)\right] d x \\
& >\int_{0}^{\bar{s}} \widetilde{F}^{i}\left(x ; \pi_{k}\right) d x=\pi \mathbb{E}\left[s_{1}\right]+(1-\pi) \mathbb{E}\left[s_{2}\right] .
\end{aligned}
$$

Hence, there exists a pool $\pi$ with $\int_{0}^{\bar{s}} \max _{i} \widetilde{F}^{i}(x ; \pi) d x>\pi \mathbb{E}\left[s_{1}\right]+(1-\pi) \mathbb{E}\left[s_{2}\right]$. Since the equilibrium is Pareto optimal, some proper pool circulates in any equilibrium.

\section{REFERENCES}

Allen, Franklin and Douglas Gale (1988), “Optimal security design.” Review of Financial Studies, 1, 229-263. [123, 124]

Biais, Bruno and Thomas Mariotti (2005), "Strategic liquidity supply and security design.” The Review of Economic Studies, 72, 615-649. [125]

Bianchi, Milo and Philippe Jehiel (2018), "Bundling, belief dispersion, and mispricing in financial markets.” Unpublished Manuscript. [123]

Broer, Tobias (2018), "Securitization bubbles: Structured finance with disagreement about default risk.” Journal of Financial Economics, 127, 505-518. [123]

Brunnermeier, Markus K. (2009), "Deciphering the liquidity and credit crunch 20072008.” Journal of Economic Perspectives, 23, 77-100. [121]

Caballero, Ricardo J. and Alp Simsek (2019) Prudential monetary policy. Technical report. National Bureau of Economic Research. [131]

Coval, Joshua, Jakub Jurek, and Erik Stafford (2009), "The economics of structured finance." Journal of Economic Perspectives, 23, 3-25. [121]

Danielsson, Jon (2008), “Blame the models.” Journal of Financial Stability, 4, 321-328. [123]

DeMarzo, Peter and Darrell Duffie (1999), “A liquidity-based model of security design.” Econometrica, 67, 65-99. [124, 125]

DeMarzo, Peter M. (2005), "The pooling and tranching of securities: A model of informed intermediation." Review of Financial Studies, 18, 1. [123, 124]

DeMarzo, Peter M., Dimitri Vayanos, and Jeffrey Zwiebel (2003), "Persuasion bias, social influence, and unidimensional opinions." The Quarterly journal of economics, 118, 909968. [123] 
Duffie, Darrell (2008), "Innovations in credit risk transfer: Implications for financial stability." [132]

Duffie, Darrell, Andreas Eckner, Guillaume Horel, and Leandro Saita (2009), "Frailty correlated default." The Journal of Finance, 64, 2089-2123. [132]

Egan, Mark L., Alexander MacKay, and Hanbin Yang (2020), Recovering Investor Expectations From Demand for Index Funds. Technical report. National Bureau of Economic Research. [123]

Ellis, Andrew and Michele Piccione (2017), “Correlation misperception in choice.” American Economic Review, 107, 1264-1292. [123]

Ellis, Andrew, Michele Piccione, and Shengxing Zhang (2019), "Equilibrium securitization with diverse beliefs." Available at SSRN 3011400. [124, 130, 136]

Enke, Benjamin and Florian Zimmermann (2017), "Correlation neglect in belief formation.” The Review of Economic Studies, 86, 313-332. [123]

Eyster, Erik and Georg Weizsäcker (2010), "Correlation neglect in financial decision making." Report. [123]

Farhi, Emmanuel and Jean Tirole (2015), “Liquid bundles.” Journal of Economic Theory, 158, 634-655. [124]

Fostel, Ana and John Geanakoplos (2012), "Tranching, cds, and asset prices: How financial innovation can cause bubbles and crashes." American Economic Journal: Macroeconomics, 4, 190-225. [123, 131]

Fostel, Ana and John Geanakoplos (2015), "Leverage and default in binomial economies: A complete characterization.” Econometrica, 83, 2191-2229. [123]

Garmaise, Mark (2001), "Rational beliefs and security design." Review of Financial Studies, 14, 1183-1213. [123, 124]

Geanakoplos, John (2001), "Liquidity, default and crashes: Endogenous contracts in general equilibrium.” [123]

Geerolf, François (2015), Leverage and Disagreement. Technical report, Working Paper. [123]

Gong, Feixue and Gregory Phelan (2016), "Debt collateralization, capital structure, and maximal leverage." [123]

Greenwood, Robin and Andrei Shleifer (2014), "Expectations of returns and expected returns." The Review of Financial Studies, 27, 714-746. [123]

Griffin, John M., Jordan Nickerson, and Dragon Yongjun Tang (2013), "Rating shopping or catering? An examination of the response to competitive pressure for cdo credit ratings." The Review of Financial Studies, 26, 2270-2310. [123, 132]

Harrison, J. Michael and David Kreps (1978), "Speculative investor behavior in a stock market with heterogeneous expectations." Quarterly Journal of Economics, 92, 323-336. http://EconPapers.repec.org/RePEc:oup:qjecon:v:92:y:1978:i:2:p:323-336. [131] 
Hellwig, Martin (2009), "Systemic risk in the financial sector: An analysis of the subprime-mortgage financial crisis." De Economist, 157, 129-208. [121]

Innes, Robert D. (1990), "Limited liability and incentive contracting with ex-ante action choices.” Journal of economic theory, 52, 45-67. [124, 125]

Levy, Gilat and Ronny Razin (2015), "Correlation neglect, voting behavior, and information aggregation.” American Economic Review, 105, 1634-1645. [123]

Lewis, Michael (2010), The Big Short. Penguin. [121]

Martin, Ian and Dimitris Papadimitriou (2019), "Sentiment and speculation in a market with heterogeneous beliefs." [131]

Meeuwis, Maarten, Jonathan A. Parker, Antoinette Schoar, and Duncan I. Simester (2018), Belief Disagreement and Portfolio Choice. Technical report. National Bureau of Economic Research. [123]

Nachman, David C. and Thomas H. Noe (1994), "Optimal design of securities under asymmetric information.” The Review of Financial Studies, 7, 1-44. [125]

Nelson, Roger B. (2006), An Introduction to Copulas, second edition. Springer. [134]

Nickerson, Jordan and John M. Griffin (2017), "Debt correlations in the wake of the financial crisis: What are appropriate default correlations for structured products?" Journal of Financial Economics, 125, 454-474. [132]

Ortner, Juan M. and Martin C. Schmalz (2016), “Disagreement and optimal security design." Unpublished. [124]

Ortoleva, Pietro and Erik Snowberg (2015), “Overconfidence in political behavior.” American Economic Review, 105, 504-535. [123]

Rubinstein, Ariel and Yuval Salant (2015), ““They do what I do”: Positive correlation in ex-post beliefs." Report. [123]

S\&P (2015), Global Methodologies and Assumptions for Corporate Cash Flow and Synthetic c.d.o.s. Technical report. [132]

Shapiro, Joel H. (2016), A Fixed-Point Farrago. Springer, Switzerland. [142]

Simsek, Alp (2013a), "Belief disagreements and collateral constraints." Econometrica, 81, 1-53. [123, 124, 130, 131]

Simsek, Alp (2013b), "Speculation and risk sharing with new financial assets." Quarterly Journal of Economics, 128, 1365-1396. [123]

Tett, Gillian (2009), Fool's Gold: How the Bold Dream of a Small Tribe at JP Morgan Was Corrupted by Wall Street Greed and Unleashed a Catastrophe. Simon and Schuster. [121]

Co-editor Thomas Mariotti handled this manuscript.

Manuscript received 13 February, 2020; final version accepted 29 April, 2021; available online 7 May, 2021. 